\title{
Genotype $\times$ environment interaction and selection for drought adaptation in sweetpotato (Ipomoea batatas [L.] Lam.) in Mozambique
}

\author{
Maria I. Andrade $\cdot$ Abdul Naico · José Ricardo • Raúl Eyzaguirre • \\ Godwill S. Makunde $\cdot$ Rodomiro Ortiz $\cdot$ Wolfgang J. Grüneberg
}

Received: 18 November 2015/Accepted: 24 March 2016/Published online: 9 April 2016

(C) The Author(s) 2016. This article is published with open access at Springerlink.com

\begin{abstract}
Sweetpotato is grown throughout the year in Mozambique but drought affects storage root yield and biomass productivity. The objectives of this research were to estimate the impact of genotype $\times$ environment interactions $(G \times E)$ in sweetpotato and select genotypes based on drought indices such as geometric mean, percent yield reduction, drought sensitivity index and harvest index. A total of 58 clones were evaluated during the dry season of 2006, 2008 and 2009. Two treatments were applied for this multi-year trial: full irrigation and without irrigation at the middle of root initiation growth stage. The field layout was a randomized complete block design
\end{abstract}

M. I. Andrade $(\square) \cdot$ A. Naico · G. S. Makunde

International Potato Center, IIAM, Av. FPLM,

2698 Maputo, Mozambique

e-mail: m.andrade@cgiar.org

J. Ricardo

Instituto de Investigação Agraria de Mozambique, Av.

FPLM, 2698 Maputo, Mozambique

R. Eyzaguirre · W. J. Grüneberg

International Potato Center, Av. La Molina,

1895 La Molina, Lima, Peru

R. Ortiz

Department of Plant Breeding, Swedish University of Agricultural Sciences, Sundsvagen 14, Box 101,

23053 Alnarp, Sweden with three replications. 'Jonathan', 'Resisto' and 'Tanzania' were the check cultivars in each treatment. Storage root and vine yields were recorded at harvest in the trials. Harvest index was computed from the yield data. The analysis of variance, regression and the additive main effects multiplicative interaction (AMMI) analyses, plus phenotypic coefficient of variation and ecovalence were used for dissecting the $\mathrm{G} \times \mathrm{E}$ and assessing the stability of each clone. Treatment, genotype $\times$ treatment and genotype $\times$ year $(G \times Y)$ interactions had highest contributions to the variation in storage root yield observed among clones. The stability of harvest index was significantly correlated with the absolute AMMI's IPCA1 and IPCA2 values for storage root yield. Cultivar performance varied within treatments. Four clones had significantly higher storage root yield $\left(\mathrm{t} \mathrm{ha}{ }^{-1}\right)$ than 'Tanzania', the best check cultivar under drought. In conclusion, storage root yield $\left(\mathrm{t} \mathrm{ha}^{-1}\right)$ was negatively affected by drought and $\mathrm{G} \times \mathrm{Y}$ interaction. Harvest index stability and the geometric mean may be key to identify clones with storage root yield stability and high storage root yield under both treatments. At least two environments should be used at early breeding stages to consider harvest index in the early breeding cycle.

Keywords Additive main effects multiplicative interaction - Biomass · Drought sensitivity index · Ecovalence $\cdot$ Regression $\cdot$ Stability $\cdot$ Storage root yield $\cdot$ Vine $\cdot$ Water deficit 


\begin{tabular}{|c|c|}
\hline \multicolumn{2}{|c|}{ Abbreviations } \\
\hline AMMI & $\begin{array}{l}\text { Additive main effects and multiplicative } \\
\text { interaction }\end{array}$ \\
\hline CIP & International Potato Center \\
\hline $\mathrm{G} \times \mathrm{E}$ & Genotype $\times$ environment interaction \\
\hline $\mathrm{HI}$ & Harvest index \\
\hline IIAM & $\begin{array}{l}\text { Instituto de Investigaçao Agrária de } \\
\text { Moçambique }\end{array}$ \\
\hline RDM & Root dry matter \\
\hline TYLD & Total storage root yield \\
\hline
\end{tabular}

\section{Introduction}

Sweetpotato provides household food security and is an important source of energy due to its ability to grow throughout the year in some areas of the sub-Saharan Africa, where it ranks among the most widely grown root crops (Andrade et al. 2009). Mozambique grew about 122,000 ha of sweetpotato with a harvest of $890,000 \mathrm{t}$ of storage roots (FAO 2015). The average storage root yield was $7.3 \mathrm{tha}^{-1}$ in 2013. Sweetpotato production doubled in Mozambique due to promotion and dissemination of orange-fleshed (OFSP) cultivars with the aim of alleviating vitamin A deficiency in the diets, and replacing maize in areas affected by frequent flood (MICOA 2005) and drought. There are two growing seasons in Mozambique: rainy, humid, hot summer (October-March) and dry, cool winter season (May-July) near perennial rivers. The summer cultivation of sweetpotato is affected by the rainfall, which is often uneven, particularly in southern Mozambique that is drier than the north of this country.

Although sweetpotato has been regarded elsewhere as a drought tolerant crop after the storage root formation and towards physiological maturity (Indira and Kabeerathumma 1988; Valenzuela et al. 2000), drought—as a result of uneven rainfall-may cause significant storage root yield loss in Mozambique (FAO 2004; MICOA 2005). There are some sweetpotato cultivars showing some adaptation to drought (Anselmo et al. 1998; Chávez et al. 2000; Ding et al. 1997; Hou et al. 1999; Maquia et al. 2013; Wang et al. 2003; Yang et al. 1999). Their adaptation depends on various mechanisms that are affected by the onset and intensity of drought. These mechanisms include drought escape due to early and rapid root development or early bulking and maturity (Yen et al. 1964; Bouwkamp 1985), drought avoidance resulting from deep rooting (Ekanayake 1990), relative water content and water use efficiency (Kelm 2000; Zhang et al. 2006), and drought tolerance relying on osmotic adjustment due to relative contents of free amino acids and soluble sugars (Zhang et al. 2003, 2004). The sweetpotato cultivar Tanzania is known for its development plasticity that remobilizes assimilates from the shoots to the storage roots under drought, while the cultivar Jonathan avoids drought due to its morphology such as small leaf size that reduces the transpiration surface. The size of the transpiring leaf and stem areas determine the amount of water loss by a plant (Monneveux and Belhassen 1996). Shoot biomass production and vine survival are necessary along with drought escape or avoidance in drought-prone areas. These two traits ensure the availability of sweetpotato planting material for the next growing season. For example, the cultivar Resisto vanished from farmers' fields after severe droughts due to few shoot biomass production and low vine survival despite its ability to escape drought in Mozambique.

The genotype $\times$ environment interaction $(\mathrm{G} \times \mathrm{E})$ significantly affect sweetpotato growth and productivity, as noted when testing bred-germplasm or cultivars under managed drought across sites in Kenya (Kivuva et al. 2014), across locations in Mozambique (Henderson et al. 1997) and Rwanda (Jannsens 1983), varying eco-geography in Perú (Grüneberg et al. 2005), or across sites and over years in South Africa (Tekalign 2007). The $\mathrm{G} \times \mathrm{E}$ causes difficulty to selection of clones with wide adaptation, which may delay the cultivar release (Rukundo et al. 2013). The $\mathrm{G} \times \mathrm{E}$ can be sub-divided within the same location on genotype $\times$ growing season $(\mathrm{G} \times \mathrm{S})$ and genotype $\times$ year $(\mathrm{G} \times \mathrm{Y})$ interactions due to variations of plantings in the rainy summer or in a dry winter, or weather changes over years, respectively.

Knowledge on the $\mathrm{G} \times \mathrm{E}$ structure can assist sweetpotato cultivar development. For example, the adaptability and stability methods were used to select stable or high-yielding sweetpotato cultivars in The Philippines (Nasayao and Saladaga 1998). Likewise, the additive main effects and multiplicative interactions (AMMI) model-which considers additive effects for genotypes and environments and multiplicative terms for $\mathrm{G} \times \mathrm{E}-$ was very useful for 
analyzing the $\mathrm{G} \times$ and stability of sweetpotato clones in multi-environment trials in Turkey (Caliskan et al. 2007). The AMMI model also provided means for identifying suitable leafy vegetable bred-germplasm in Malaysia (Thiyagu et al. 2013) that can be further used for a new cycle of crossing and selection.

The aim of this research was to determine $\mathrm{G} \times \mathrm{E}$ patterns-particularly $\mathrm{G} \times \mathrm{S}$ for storage root yield and dry matter content — establish the magnitude of $\mathrm{G} \times \mathrm{E}$ interaction in sweetpotato under irrigation and drought, and assess the usefulness of a drought intensity index to identify suitable clones with storage root and vine yields for drought-prone environments in Mozambique. This research was also the first to study the value of sweetpotato landraces from Mozambique under drought.

\section{Materials and methods}

A total of 58 sweetpotato were evaluated at Umbeluzi Research Station over three dry seasons (Table 1). The materials comprised 40 farmer landraces (37 from Mozambique, 1 from Kenya, 1 from Tanzania and 1 from Zimbabwe) and 18 clones coming from seven different breeding programs. Farmer landraces were predominantly spreading except five that had a semierect plant type. The check cultivars were Jonathan, Resisto and Tanzania.

The clones were evaluated at Umbeluzi Research Station during the dry seasons of 2006, 2008 and 2009. Umbeluzi Research Station (12 m.a.s.l; latitude: $26^{\circ} 03^{\prime} \mathrm{S}$ and longitude: $32^{\circ} 23^{\prime} \mathrm{E}$ ) is a representative site for southern African lowlands of the tropical savanna climate at the border of the hot semi-arid climate and the humid subtropical climate zone (Kottek et al. 2006; Fig. 1). Drought is experienced on annual basis in Gaza, Inhambane and Maputo provinces as well as half of Tete province, which translates into $35 \%$ of Mozambique's area suffering from water deficit annually. The soil type at Umbeluzi Research Station is alluvial with a texture ranging from sandy loam in the topsoil to sandy at $1.75 \mathrm{~m}$ soil depth. The available water capacity from topsoil to $1.75 \mathrm{~m}$ soil depth is about $200 \mathrm{~mm}$. The main weather features and amount of irrigation water applied during growing period and evaluation in 2006, 2008 and 2009 are given in Table 2.

Two irrigation treatments were established: wellwatered and water deficit. Trials were established on 7th March 2006, 2nd June 2008 and 8th April 2009. Both treatments received four furrow irrigations of $60 \mathrm{~mm}$ each: 1 day before, and 4, 15 and 25 days after planting. The well-watered treatment received additional furrow irrigations of $40 \mathrm{~mm}$ of water at 35, 45 and 55 days after planting. Irrigation was resumed 60 days after planting in the water-deficit treatment. The well-watered treatment represented Central and Northern regions of Mozambique that receive adequate rainfall for sweetpotato production. The drought treatment mimics drought as noted in southern Mozambique. Maquia et al. (2013) rated Umbeluzi as a suitable site for screening sweetpotato germplasm under drought.

Each clone was planted in two row plots, replicated three times in each treatment following a randomized complete block design. Each row was planted with 12 plants with a plant-to-plant spacing of $0.3 \mathrm{~m}$, while the distance between rows was $0.9 \mathrm{~m}$. The previous crop to all experiments was maize, which received $92 \mathrm{~kg} \mathrm{~N} \mathrm{ha}^{-1}$ from urea. The irrigation treatments were applied to an entire block in particular three blocks received well-watered treatment and three blocks were under water deficit in each season. Hoe weeding was employed to keep weed-freed plots, which did not receive either synthetic fertilizers or pesticides.

Harvesting was done on 10th August 2006, 23rd November 2008 and 17th September 2009. At harvest 10 plants were taken from each plot for recording storage root $\left(\mathrm{t} \mathrm{ha}^{-1}\right)$, vine yields $\left(\mathrm{t} \mathrm{ha}^{-1}\right)$ and harvest index $(\%)$. Five roots of medium size were randomly collected from each plot to determine dry matter (DM) content in the laboratory. Each root from each laboratory sample was washed to remove soil particles and rinsed with abundant tap water, peeled and cut longitudinally into four sections. Two opposite sections of each root were used to prepare a $100 \mathrm{~g}$ compound sample that was weighed and placed in paper bags and oven dried for $72 \mathrm{~h}$. After the drying period, the samples were reweighed again to determine DM content. Drought stress indices for storage root yield and vine yield were computed (Agili et al. 2012).

The geometric mean productivity is the square root of the product of storage root yield under drought and storage root yield under irrigated condition $(\mathrm{GMP})=\sqrt{ }$ storage root yield under drought $\times$ storage root yield under irrigated condition (Ramirez-Vallejo and Kelly 1998). 
Table 1 Name, code by the International Potato Center (CIP) or Instituto de Investigaçao Agrária de Moçambique (IIAM), cultivar type (CT), country of origin (CO), storage root traits and plant type of sweetpotato landraces and bred-germplasm included in multi-environment testing under irrigation and nonirrigation plots at Umbeluzi (Mozambique) between 2006 and 2009

\begin{tabular}{|c|c|c|c|c|c|c|c|c|}
\hline \multirow[t]{2}{*}{ Name } & \multirow[t]{2}{*}{ CIP code } & \multirow[t]{2}{*}{ IIAM code } & \multirow[t]{2}{*}{$\mathrm{CT}^{\mathrm{a}}$} & \multirow[t]{2}{*}{$\mathrm{CO}^{\mathrm{b}}$} & \multicolumn{4}{|l|}{ Storage root } \\
\hline & & & & & Skin color ${ }^{\mathrm{c}}$ & Flesh color $^{\mathrm{d}}$ & Shape ${ }^{\mathrm{e}}$ & Plant type $^{f}$ \\
\hline Tacna & 187019.1 & MZC0001 & BL & $\mathrm{PE}$ & $\mathrm{BO}$ & DY & $\mathrm{RE}$ & SE \\
\hline Chissicuana-2 & NA & MZC0002 & FV & $\mathrm{MZ}$ & $\mathrm{BO}$ & LY & $\mathrm{RE}$ & $\mathrm{S}$ \\
\hline Nhacutse-5 & NA & MZC0003 & FV & $\mathrm{MZ}$ & $\mathrm{BO}$ & DY & LE & $\mathrm{S}$ \\
\hline Nwaracu & NA & MZC0004 & FV & $\mathrm{MZ}$ & DR & DY & $\mathrm{E}$ & $\mathrm{S}$ \\
\hline Nwazambane & NA & MZC0005 & FV & $\mathrm{MZ}$ & $\mathrm{C}$ & LY & $\mathrm{E}$ & $\mathrm{S}$ \\
\hline NASPOT5 & NA & MZC0006 & MV & UG & $\mathrm{BO}$ & IO & $\mathrm{RE}$ & $\mathrm{S}$ \\
\hline Malawe & NA & MZC0007 & FV & $\mathrm{MZ}$ & $\mathrm{R}$ & LO & $\mathrm{O}$ & $\mathrm{S}$ \\
\hline Nhacoongo-1 & NA & MZC0008 & $\mathrm{FV}$ & $\mathrm{MZ}$ & $\mathrm{C}$ & $\mathrm{W}$ & LE & $\mathrm{S}$ \\
\hline Mamphenane & NA & MZC0009 & MV & SA & $\mathrm{BO}$ & LO & OBL & $\mathrm{S}$ \\
\hline Maphuta & NA & MZC0010 & MV & SA & $\mathrm{P}$ & DY & $\mathrm{E}$ & $\mathrm{E}$ \\
\hline Nwamanhiça & NA & MZC0011 & $\mathrm{FV}$ & $\mathrm{MZ}$ & $\mathrm{C}$ & $\mathrm{Y}$ & $\mathrm{E}$ & $\mathrm{S}$ \\
\hline 199062.1 & 199062.1 & MZC0012 & BL & $\mathrm{PE}$ & $\mathrm{BO}$ & IO & $\mathrm{RE}$ & $\mathrm{S}$ \\
\hline Nhacutse-3 & NA & MZC0013 & $\mathrm{FV}$ & $\mathrm{MZ}$ & $\mathrm{C}$ & $\mathrm{C}$ & $\mathrm{RE}$ & $\mathrm{S}$ \\
\hline ADMARC & NA & MZC0014 & $\mathrm{FV}$ & $\mathrm{MZ}$ & $\mathrm{P}$ & $\mathrm{C}$ & $\mathrm{E}$ & $\mathrm{S}$ \\
\hline Diliva & NA & MZC0015 & FV & $\mathrm{MZ}$ & $\mathrm{P}$ & $\mathrm{Y}$ & $\mathrm{RE}$ & SE \\
\hline ST87-030 & 189001.2 & MZC0016 & BL & CIP & $\mathrm{Y}$ & $\mathrm{Y}$ & $\mathrm{RE}$ & SE \\
\hline 440203 & 440203 & MZC0017 & MV & CIP & $\mathrm{BO}$ & $\mathrm{W}$ & OBV & $\mathrm{S}$ \\
\hline Thuang-Thuang & NA & MZC0018 & $\mathrm{FV}$ & $\mathrm{MZ}$ & $\mathrm{BO}$ & W & $\mathrm{RE}$ & $\mathrm{S}$ \\
\hline Atacama & 187020.1 & MZC0019 & MV & $\mathrm{PE}$ & $\mathrm{DR}$ & $\mathrm{Y}$ & $\mathrm{RE}$ & $\mathrm{S}$ \\
\hline $1998-12-3$ & NA & MZC0020 & $\mathrm{BL}$ & PE & LB & DO & $\mathrm{E}$ & $\mathrm{S}$ \\
\hline Chissicuana-3 & NA & MZC0021 & $\mathrm{FV}$ & $\mathrm{MZ}$ & $\mathrm{C}$ & $\mathrm{W}$ & $\mathrm{RE}$ & $\mathrm{S}$ \\
\hline Nhacutse-1 & NA & MZC0022 & FV & $\mathrm{MZ}$ & $\mathrm{C}$ & $\mathrm{Y}$ & LO & $\mathrm{S}$ \\
\hline Canassumana & NA & MZC0023 & FV & $\mathrm{MZ}$ & $\mathrm{R}$ & $\mathrm{Y}$ & $\mathrm{E}$ & $\mathrm{S}$ \\
\hline UNK-Malawe & NA & MZC0024 & FV & $\mathrm{MZ}$ & $\mathrm{R}$ & $\mathrm{Y}$ & $\mathrm{O}$ & $\mathrm{S}$ \\
\hline Nhacutse-2 & NA & MZC0025 & $\mathrm{FV}$ & $\mathrm{MZ}$ & $\mathrm{C}$ & $\mathrm{W}$ & $\mathrm{RE}$ & $\mathrm{S}$ \\
\hline Chitandzana & NA & MZC0026 & $\mathrm{FV}$ & $\mathrm{MZ}$ & $\mathrm{R}$ & LY & LE & $\mathrm{S}$ \\
\hline Jogó & NA & MZC0027 & $\mathrm{FV}$ & $\mathrm{MZ}$ & $\mathrm{P}$ & LY & $\mathrm{R}$ & $\mathrm{S}$ \\
\hline Xiadlaxakau & NA & MZC0028 & $\mathrm{FV}$ & $\mathrm{MZ}$ & $\mathrm{R}$ & $\mathrm{Y}$ & $\mathrm{R}$ & $\mathrm{S}$ \\
\hline Xitsekele & NA & MZC0029 & $\mathrm{FV}$ & $\mathrm{MZ}$ & $\mathrm{P}$ & $\mathrm{Y}$ & $\mathrm{E}$ & $\mathrm{S}$ \\
\hline Chissicuana-1 & NA & MZC0030 & FV & $\mathrm{MZ}$ & DR & DY & $\mathrm{RE}$ & $\mathrm{S}$ \\
\hline Nhacutse-4 & NA & MZC0031 & $\mathrm{FV}$ & $\mathrm{MZ}$ & $\mathrm{BO}$ & $\mathrm{W}$ & $\mathrm{E}$ & SE \\
\hline Jogó-2 & NA & MZC0032 & FV & $\mathrm{MZ}$ & $\mathrm{BO}$ & $\mathrm{W}$ & $\mathrm{E}$ & $\mathrm{S}$ \\
\hline Manhissane & NA & MZC0033 & $\mathrm{FV}$ & $\mathrm{MZ}$ & $\mathrm{C}$ & W & $\mathrm{E}$ & $\mathrm{S}$ \\
\hline Nwamazambe & NA & MZC0034 & FV & $\mathrm{MZ}$ & $\mathrm{BO}$ & $\mathrm{Y}$ & $\mathrm{E}$ & $\mathrm{S}$ \\
\hline Mafambane & NA & MZC0035 & $\mathrm{FV}$ & $\mathrm{MZ}$ & $\mathrm{C}$ & W & OBV & $\mathrm{S}$ \\
\hline Nwamonguane & NA & MZC0036 & $\mathrm{FV}$ & $\mathrm{MZ}$ & $\mathrm{C}$ & $\mathrm{C}$ & $\mathrm{E}$ & $\mathrm{S}$ \\
\hline Chulamete & NA & MZC0037 & $\mathrm{FV}$ & $\mathrm{MZ}$ & $\mathrm{C}$ & $\mathrm{Y}$ & $\mathrm{RE}$ & $\mathrm{S}$ \\
\hline Cinco minutos & NA & MZC0038 & $\mathrm{FV}$ & $\mathrm{MZ}$ & $\mathrm{C}$ & $\mathrm{Y}$ & $\mathrm{RE}$ & $\mathrm{S}$ \\
\hline Xiphone & NA & MZC0039 & $\mathrm{FV}$ & $\mathrm{MZ}$ & $\mathrm{C}$ & $\mathrm{W}$ & $\mathrm{RE}$ & $\mathrm{S}$ \\
\hline Nwaxitsimbwane & NA & MZC0040 & FV & $\mathrm{MZ}$ & $\mathrm{R}$ & $\mathrm{W}$ & $\mathrm{RE}$ & SE \\
\hline Cacilda & NA & MZC0041 & $\mathrm{FV}$ & $\mathrm{MZ}$ & $\mathrm{C}$ & $\mathrm{W}$ & OBV & $\mathrm{S}$ \\
\hline
\end{tabular}


Table 1 continued

\begin{tabular}{|c|c|c|c|c|c|c|c|c|}
\hline \multirow[t]{2}{*}{ Name } & \multirow[t]{2}{*}{ CIP code } & \multirow[t]{2}{*}{ IIAM code } & \multirow[t]{2}{*}{$\mathrm{CT}^{\mathrm{a}}$} & \multirow[t]{2}{*}{$\mathrm{CO}^{\mathrm{b}}$} & \multicolumn{4}{|l|}{ Storage root } \\
\hline & & & & & Skin color ${ }^{\mathrm{c}}$ & Flesh color $^{\mathrm{d}}$ & Shape $e^{e}$ & Plant type $^{\mathrm{f}}$ \\
\hline Nwanaqtsjo & NA & MZC0042 & $\mathrm{FV}$ & $\mathrm{MZ}$ & $\mathrm{C}$ & $\mathrm{C}$ & $\mathrm{E}$ & $\mathrm{S}$ \\
\hline Ligodo & NA & MZC0043 & $\mathrm{FV}$ & $\mathrm{MZ}$ & $\mathrm{C}$ & $\mathrm{C}$ & $\mathrm{RE}$ & SE \\
\hline Xihetamakote & NA & MZC0044 & $\mathrm{FV}$ & $\mathrm{MZ}$ & $\mathrm{C}$ & DY & $\mathrm{O}$ & S \\
\hline TIS 9265 & 440075 & MZC0045 & $\mathrm{BL}$ & NG & LB & W & OBV & $\mathrm{S}$ \\
\hline Ximitakwatse & NA & MZC0046 & $\mathrm{FV}$ & $\mathrm{MZ}$ & $\mathrm{C}$ & $\mathrm{Y}$ & $\mathrm{O}$ & $\mathrm{S}$ \\
\hline Resisto & 440001 & MZC0047 & MV & US & $\mathrm{R}$ & DO & $\mathrm{RE}$ & SE \\
\hline Jonathan & 420014 & MZC0048 & MV & $\mathrm{PE}$ & BO & IO & $\mathrm{O}$ & $\mathrm{E}$ \\
\hline Japon Tremesino Selecto & 420009 & MZC0049 & MV & $\mathrm{PE}$ & BO & IO & $\mathrm{O}$ & $\mathrm{E}$ \\
\hline $\mathrm{CN}-448-49$ & 440181 & MZC0050 & MV & TW & $\mathrm{BO}$ & $\mathrm{IO}$ & $\mathrm{O}$ & $\mathrm{E}$ \\
\hline Tainung-64 & 440189 & MZC0051 & MV & TW & $\mathrm{BO}$ & DO & $\mathrm{RE}$ & $\mathrm{S}$ \\
\hline Cordner & NA & MZC0052 & MV & US & $\mathrm{BO}$ & DO & $\mathrm{RE}$ & SE \\
\hline Tanzania & NA & MZC0053 & & $\mathrm{TZ}$ & $\mathrm{C}$ & $\mathrm{Y}$ & $\mathrm{O}$ & SE \\
\hline TIS-2534 & 44006 & MZC0054 & $\mathrm{BL}$ & NG & $\mathrm{R}$ & W & $\mathrm{O}$ & $\mathrm{S}$ \\
\hline $\mathrm{MgCl} 01$ & NA & MZC0055 & FV & $\mathrm{MZ}$ & $\mathrm{C}$ & DO & $\mathrm{RE}$ & $\mathrm{S}$ \\
\hline Moz-White & NA & MZC0056 & $\mathrm{FV}$ & ZW & $\mathrm{R}$ & W & $\mathrm{E}$ & $\mathrm{S}$ \\
\hline Lo-323 & 440185 & MZC0057 & BL & US & $\mathrm{BO}$ & DO & $\mathrm{O}$ & $\mathrm{E}$ \\
\hline SPK004 & 441768 & MZC0058 & FV & $\mathrm{KE}$ & $\mathrm{C}$ & IO & $\mathrm{RE}$ & SE \\
\hline
\end{tabular}

${ }^{a} C T$ cultivar type, $F V$ farmers' landrace, $B L$ breeding clone, $M V$ modern cultivar

b $C O$ country of origin, $J P$ Japan, $K E$ Kenya, $M Z$ Mozambique, $N G$ Nigeria, $P E$ Perú, $S A$ South Africa, $T Z$ Tanzania, $T W$ Taiwan, $U S$ United States of America, ZW Zimbabwe

c $C$ cream, $L B$ light brown, $B O$ brown orange, $R$ red, $D R$ dark red, $P$ pink

${ }^{\mathrm{d}} W$ white, $L$ light yellow, $I Y$ intermediate yellow, $D Y$ dark yellow, $C$ cream, $L O$ light orange, $I O$ intermediate orange, $D O$ dark orange

e $R E$ round elliptic, $E$ elliptic, $L E$ long elliptic, $O B V$ obovate, $O B L$ oblong

f $E$ erect, $S E$ semi-erect, $S$ spreading

Drought intensity index (DII) and drought susceptibility index (DSI) were calculated by formula given by Fisher and Maurer (1978) as follows: DII = 1 - mean storage root yield of all clones under drought/mean storage root yield of all clones under irrigated condition, and DSI $=(1-$ storage root yield under drought/storage root yield under irrigation)/DII. Drought tolerance efficiency (DTE) was calculated by formula given by Fischer and Wood (1981); i.e., DTE \% = (yield under drought/ yield under irrigation) $\times 100$. Percent reduction $(P R)$ was also computed for storage root yield. $\mathrm{PR}=[$ (yield under irrigation - yield under drought)/yield under irrigation] $\times 100$.

Storage root and vine yields $\left(\mathrm{t} \mathrm{ha}^{-1}\right)$, DM (\%) and harvest index data were subjected to statistical analyses in Plant Breeding Statistical Program (PLABSTAT; Utz 1997), SAS 6.12 (SAS Institute 1988, 1997) and R ( $R$ core team 2015). The data were analyzed in four steps. In the first step phenotypic data were analyzed separately for each treatment in each season using the analysis of variance (ANOVA) for the randomized complete bock design with SAS 6.12 GLM procedure. In the second step phenotypic data were analyzed across treatments and seasons using PLABSTAT and the model statement $\mathrm{G}+\mathrm{T}+\mathrm{S}+$ $\mathrm{GT}+\mathrm{GS}+\mathrm{TS}+\mathrm{GTS}+\mathrm{R}: \mathrm{TS}+\mathrm{RGTS}$, where G corresponds to genotypes, $\mathrm{T}$ to treatments and $\mathrm{S}$ to seasons. In this model we considered all the main effects $(\mathrm{G}, \mathrm{T}$, and $\mathrm{S})$ the interactions among genotypes, treatments, and seasons (GT, GS, TS, and GTS), and the effect of blocks nested into treatments and seasons (R:TS). The term RGTS corresponds to the error term. In the third step, each combination between treatments and seasons was considered an environment (E) for the estimation of stability and adaptability of clones. The dynamic concept of stability was applied for all the 


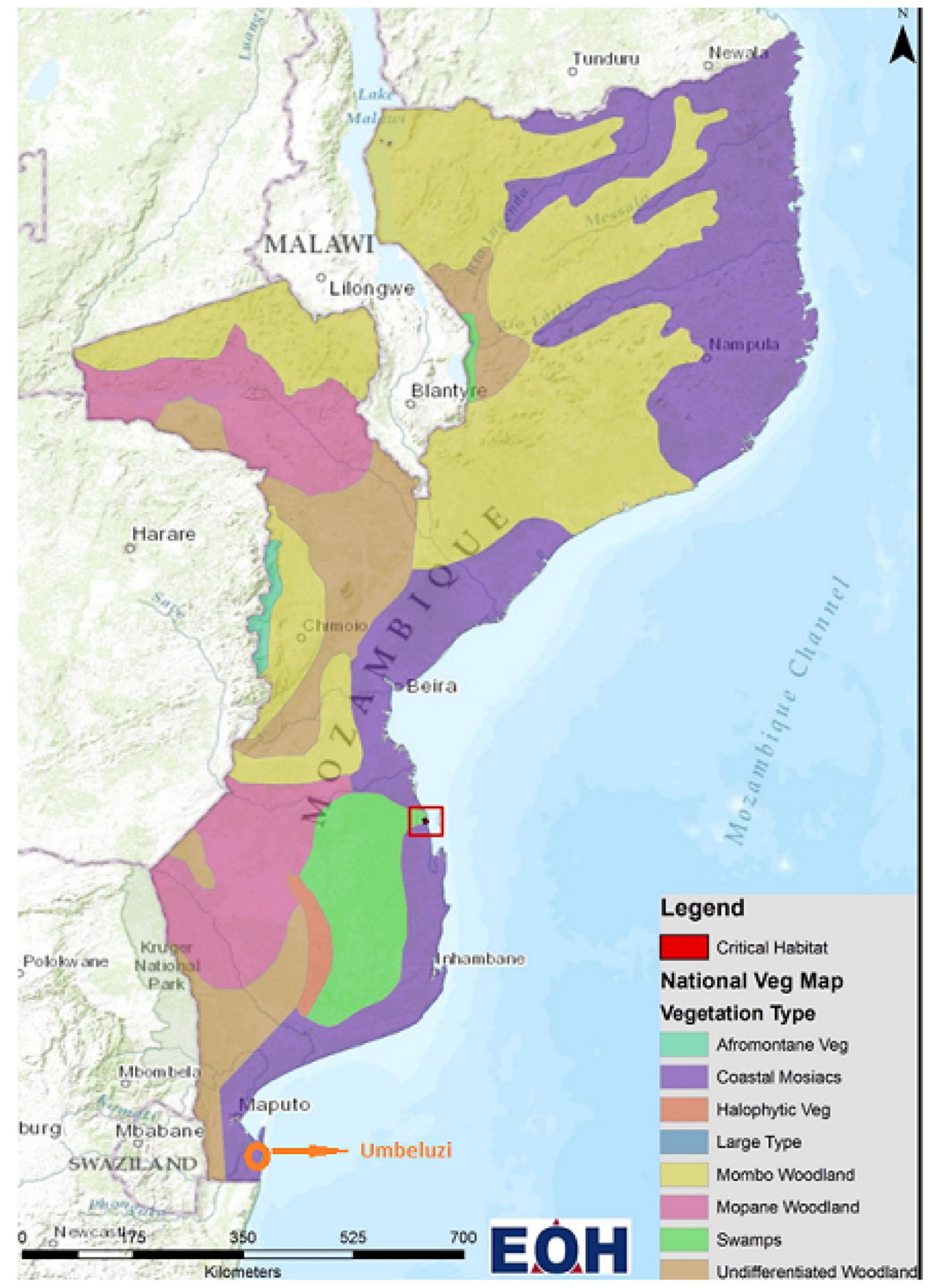

Fig. 1 Position of Umbeluzi Research Station and vegetation distribution in Mozambique 
Table 2 Average monthly rainfall $(\mathrm{R}, \mathrm{mm})$, temperature $\left(\mathrm{T},{ }^{\circ} \mathrm{C}\right)$, evapotranspiration $(\mathrm{ET})$, water applied in irrigated (IRR, mm) and drought $(\mathrm{DR}, \mathrm{mm})$ treatments during growing period of multi-environment trials of sweetpotato at Umbeluzi

\begin{tabular}{lllllllllll}
\hline Year & MP $^{\mathrm{a}}$ & March & April & May & June & July & August & September & October & November \\
\hline 2006 & $\mathrm{R}$ & 163.7 & 22.7 & 1.5 & 3.6 & 8.3 & 18.8 & - & - & - \\
& $\mathrm{T}$ & 24.9 & 23.3 & 20.5 & 19.2 & 19.7 & 20.4 & & & - \\
IRR & & - & 160 & 160 & 160 & 120 & 60 & - & - & - \\
DR & & - & 60 & 80 & 80 & 60 & 40 & - & - & - \\
& ET & 139.1 & 111.5 & 126.3 & 110.4 & 116.0 & 156.2 & - & - & - \\
2008 & R & - & - & - & 14.2 & 0 & 0 & 13.3 & 0.5 & 128.7 \\
& T & & & & 19.6 & 20 & 21.6 & 22.9 & 24.2 & 25.7 \\
IRR & & - & - & - & 160 & 160 & 160 & 160 & 120 & - \\
DR & & - & - & - & 160 & 60 & 80 & 80 & 120 & - \\
& ET & - & - & - & 94.1 & 125.7 & 168.6 & 195.9 & 200.6 & 166.0 \\
2009 & $\mathrm{R}$ & - & 5.4 & 1.7 & 13.0 & 5.5 & 61.9 & 0 & - & - \\
& T & & 23.6 & 22.0 & 20.5 & 18.5 & 20.1 & 22.5 & & - \\
IRR & & - & 160 & 160 & 160 & 160 & 60 & 60 & - & - \\
DR & & - & 160 & 60 & 60 & 60 & 60 & 60 & - & - \\
& ET & - & 124.4 & 113.6 & 109.4 & 150.0 & 133.3 & 130.9 & - & - \\
\hline
\end{tabular}

${ }^{a}$ Meteorological parameter

yield traits where a stable genotype has a varying yield response to the tested genotypes (Becker and Leon 1988). This concept recognizes a specific set of tested genotypes and equals Type II stability with high goodness of fit (Lin et al. 1986). The ecovalence technique was also used to estimate trait stability (Wricke 1962). The phenotypic coefficient of variation across environments (CV) was calculated for each sweetpotato clone (Francis and Kannenberg 1978). In a fourth step the AMMI model (Gollob 1968; Gauch 1992) was fitted using $R$ to decompose the $G \times E$ matrix of interaction effects; the first two principal components were presented bi-plots. Finally, correlations among stability parameters the principal component scores of the AMMI analysis were carried out for harvest index (\%) and storage root yield $\left(\mathrm{t} \mathrm{ha}^{-1}\right)$ by SAS procedure CORR and the optional statement PEARSON.

\section{Results}

There were significant differences for storage root yield, vine yield and total biomass between the two treatments, among genotypes, and due to the $\mathrm{G} \times \mathrm{E}$ (Table 3 ). The $\mathrm{G} \times \mathrm{E}$ variation was also greater than the environmental or genotypic effects for storage root yield. Both components of the $\mathrm{G} \times \mathrm{E}$ variation, viz. heterogeneity among regression lines and the remainder (deviation) were significant (Table 3 ). The significant linear $\mathrm{G} \times \mathrm{E}$ indicated the possibility of predicting trait performance of individual genotypes from the linear regression across environments.

Drought affected total biomass, storage root yield and vine across testing years (Table 4). The storage root yields among the landraces, cultivars and other bred-germplasm ranged from 2.24 to $17.38 \mathrm{t} \mathrm{ha}^{-1}$ under irrigation and 1.4 to $14.72 \mathrm{t} \mathrm{ha}^{-1}$ without irrigation (Table 4). Modern cultivars and other bredgermplasm had higher storage root yields under both treatments than the farmers' landraces. The storage root yields of Chissicuana-2, ADMARC, ThuangThuang, Nhacutse-2, Xiadlaxakau, Nwamazambe, Cacilda, Ligodo, Ximitakwatse, Tacna, Mamphenane, 199062.1, ST87-030, Atacama, 1998-12-3, and TIS2534 were above that of the best cultivar check Resisto under irrigation.

Edible yield is often used to assess adaptation to drought among genotypes (Blum 2005). The storage root yield of ADMARC, Chissicuana-2, Tacna, TIS2534 and Xiadlaxakau - among the high yielding under irrigation- plus UNK-Malawe, Xitsekele, and Chulamete was above that of Tanzania (=Chingova), which was the best cultivar check under drought 
Table 3 Multi-environment analysis of variance including heterogeneity due to regression (Het. R.), deviations from regression lines (Dev. R.), stability variance $\left(\sigma^{2}\right)$ and relative variance (Rel. $\sigma^{2}$ ) for storage root yield, vine yield and biomass

\begin{tabular}{|c|c|c|c|c|c|}
\hline Trait & Source of variation & Degrees of freedom & Mean square & $\sigma^{2}$ & Rel. $\sigma^{2}$ \\
\hline \multirow[t]{8}{*}{ Storage root yield } & Environment (E) & 5 & 1154.682 & $5.801 * *$ & 98.24 \\
\hline & Genotype (G) & 57 & 158.178 & $5.905 * *$ & 100 \\
\hline & $\mathrm{G} \times \mathrm{E}$ & 285 & 51.894 & $12.904 * *$ & 218.54 \\
\hline & Het. R. & 57 & 72.722 & $1.446 * *$ & $11.21^{\mathrm{a}}$ \\
\hline & Dev. R. & 228 & 46.687 & $11.169 * *$ & $86.55^{\mathrm{a}}$ \\
\hline & Het. R. & 5 & 240.096 & $1.101 * *$ & $8.53^{\mathrm{a}}$ \\
\hline & Dev. R. & 280 & 48.533 & $11.784 * *$ & $91.32^{\mathrm{a}}$ \\
\hline & Error & 596 & 13.181 & 13.181 & 223.22 \\
\hline \multirow[t]{8}{*}{ Vine yield } & $\mathrm{E}$ & 5 & 7162.892 & $36.007 * *$ & 60.64 \\
\hline & G & 57 & 1257.096 & $59.375 * *$ & 100 \\
\hline & $\mathrm{G} \times \mathrm{E}$ & 285 & 188.341 & $37.732 * *$ & 63.54 \\
\hline & SUB Het. R.G & 57 & 422.264 & $16.245 * *$ & $43.05^{\mathrm{a}}$ \\
\hline & Dev. R.G & 228 & 129.860 & $18.239 * *$ & $48.34^{\mathrm{a}}$ \\
\hline & SUB Het. R.E & 5 & 3086.723 & $16.955^{* *}$ & $44.94^{\mathrm{a}}$ \\
\hline & Dev. R.E & 280 & 136.584 & $20.480 * *$ & $54.28^{\mathrm{a}}$ \\
\hline & Error & 621 & 75.144 & 75.144 & 126.55 \\
\hline \multirow[t]{8}{*}{ Biomass } & $\mathrm{E}$ & 5 & 8577.841 & $41.256 * *$ & 65.74 \\
\hline & G & 57 & 1407.432 & $62.748 * *$ & 100 \\
\hline & $\mathrm{G} \times \mathrm{E}$ & 285 & 277.965 & $59.917 * *$ & 95.48 \\
\hline & Het. R. & 57 & 423.098 & $10.079 * *$ & $16.82^{\mathrm{a}}$ \\
\hline & Dev. R. & 228 & 241.682 & $47.823 * *$ & $79.82^{\mathrm{a}}$ \\
\hline & Het. R. & 5 & 3671.485 & $19.851 * *$ & $33.13^{\mathrm{a}}$ \\
\hline & Dev. R. & 280 & 217.367 & $39.718 * *$ & $66.29^{\mathrm{a}}$ \\
\hline & Error & 606 & 98.214 & 98.214 & 156.52 \\
\hline
\end{tabular}

** Indicates and highly significant at $P \leq 0.01$

${ }^{\text {a }}$ Relative to $\sigma_{\mathrm{G} \times \mathrm{E}}^{2}$

(Table 4). The storage root yield of the check cultivar Tanzania was higher under drought than under irrigation but the reverse was true for its vine and biomass yield, which were high under irrigation. ADMARC, Tacna and Xiadlaxakau had the best performance without irrigation; their storage root yield was at least $10 \mathrm{t} \mathrm{ha}^{-1}$.

The degree of drought imposed on the trials across the 3 years was moderate (DII $=0.25$ ). Based on the drought stress indices, ADMARC and Tacna were rated as drought tolerant (Table 5) and appeared on one cluster (Fig. 2). Their storage root yields were above $10 \mathrm{tha}^{-1}$ with or without irrigation, while their DTE was above $75 \%$ and the DSI was below 1 . Both cultivars kept a high harvest of sweetpotato landraces and bred-germplasm included in multi-environment testing under irrigation and non-irrigation plots at Umbelezi (Mozambique) between 2006 and 2009 
Table 4 Estimates obtaining using the dynamic concept of genotype $\times$ environment interaction $\left(\mathrm{x}_{\mathrm{i}}\right.$ : mean, $\beta$ : regression coefficient: MS: mean square) and the ecovalence (Eco) for storage root yield, vine yield and biomass for environments and genotypes (Instituto de Investigaçao Agrária de Moçambique [IIAM] code-Table 1) of sweetpotato included in multienvironment testing under irrigation (IR) and non-irrigation (NI) plots at Umbelezi (Mozambique) between 2006 and 2009

\begin{tabular}{|c|c|c|c|c|c|c|c|c|c|c|c|c|}
\hline & \multicolumn{4}{|c|}{ Storage root yield } & \multicolumn{4}{|c|}{ Vine yield } & \multicolumn{4}{|c|}{ Biomass } \\
\hline & $\mathrm{x}_{\mathrm{i}}$ & $\beta$ & MS & Eco & $\mathrm{x}_{\mathrm{i}}$ & $\beta$ & MS & Eco & $\mathrm{x}_{\mathrm{i}}$ & $\beta$ & MS & Eco \\
\hline \multicolumn{13}{|c|}{ Environments } \\
\hline IR-2006 & 5.2 & 1.13 & 10.9 & 10.9 & 26.5 & 1.49 & 27.4 & 43.8 & 31.7 & 1.51 & 48.8 & 68.1 \\
\hline NI-2006 & 4.9 & 1.07 & 15.3 & 15.1 & 21.2 & 1.55 & 48.5 & 68.6 & 26.0 & 1.57 & 82.8 & 106.8 \\
\hline IR-2008 & 7.1 & 0.95 & 8.6 & 8.4 & 23.9 & 1.33 & 52.5 & 59.2 & 31.0 & 1.33 & 62.1 & 69.6 \\
\hline NI-2008 & 2.3 & 0.30 & 2.3 & 6.6 & 10.9 & 0.53 & 32.7 & 47.5 & 13.2 & 0.52 & 41.5 & 58.8 \\
\hline IR-2009 & 9.0 & 1.53 & 23.8 & 25.8 & 17.8 & 0.64 & 47.5 & 55.8 & 26.4 & 0.63 & 82.6 & 91.8 \\
\hline NI-2009 & 8.7 & 1.03 & 20.1 & 19.8 & 11.6 & 0.46 & 19.0 & 39.0 & 20.2 & 0.44 & 44.4 & 68.2 \\
\hline $\mathrm{LSD}_{0 . .05}$ & & 0.48 & & & & 0.28 & & & & 0.35 & & \\
\hline$\beta$-test & & & $* *$ & & & & + & & & & $*$ & \\
\hline \multicolumn{13}{|l|}{ Genotypes } \\
\hline MZC0001 & 15.6 & 3.20 & 12.2 & 41.9 & 18.2 & 1.23 & 43.0 & 36.5 & 33.8 & 1.25 & 60.9 & 51.8 \\
\hline MZC0002 & 11.2 & 1.51 & 28.0 & 24.2 & 34.6 & 2.49 & 134.0 & 198.9 & 45.8 & 2.04 & 138.1 & 163.7 \\
\hline MZC0003 & 6.3 & 0.97 & 2.1 & 1.7 & 28.3 & 2.20 & 13.0 & 69.2 & 34.6 & 1.85 & 25.8 & 56.4 \\
\hline MZC0004 & 3.4 & -0.28 & 8.1 & 17.3 & 17.8 & 1.55 & 22.3 & 30.1 & 21.2 & 1.49 & 79.1 & 75.0 \\
\hline MZC0005 & 6.8 & 1.58 & 13.1 & 12.9 & 15.5 & 0.36 & 16.8 & 30.4 & 22.2 & 0.15 & 33.2 & 62.1 \\
\hline MZC0006 & 4.5 & 1.03 & 10.6 & 8.5 & 31.9 & 2.70 & 125.9 & 218.8 & 36.4 & 2.00 & 156.6 & 174.6 \\
\hline MZC0007 & 5.0 & 1.34 & 6.4 & 5.8 & 14.5 & -0.40 & 27.3 & 102.7 & 19.4 & -0.09 & 89.3 & 129.6 \\
\hline MZC0008 & 3.4 & 0.21 & 6.9 & 9.7 & 31.1 & 1.84 & 4.0 & 32.5 & 34.4 & 1.86 & 30.4 & 60.3 \\
\hline MZC0009 & 7.3 & 1.26 & 11.3 & 9.5 & 6.4 & 0.30 & 32.0 & 45.8 & 13.5 & 0.55 & 56.4 & 54.9 \\
\hline MZC0010 & 4.6 & 0.64 & 11.9 & 10.4 & 10.3 & -0.05 & 0.9 & 46.2 & 14.7 & 0.00 & 18.5 & 64.5 \\
\hline MZC0011 & 2.6 & 0.58 & 3.0 & 3.6 & 19.4 & 1.15 & 97.4 & 78.8 & 22.8 & 1.08 & 134.6 & 108.0 \\
\hline MZC0012 & 11.5 & 0.22 & 51.7 & 45.4 & 15.7 & 1.54 & 2.5 & 14.0 & 27.2 & 2.03 & 50.9 & 92.8 \\
\hline MZC0013 & 3.9 & 0.74 & 4.8 & 4.3 & 23.5 & 1.10 & 50.5 & 40.8 & 27.4 & 0.99 & 63.5 & 50.8 \\
\hline MZC0014 & 12.0 & 0.04 & 42.3 & 40.0 & 23.3 & 2.30 & 61.2 & 117.8 & 35.6 & 2.61 & 199.4 & 286.6 \\
\hline MZC0015 & 2.4 & 0.43 & 2.0 & 3.7 & 37.3 & 1.57 & 64.2 & 64.9 & 39.0 & 1.19 & 110.8 & 90.5 \\
\hline MZC0016 & 9.1 & 2.08 & 8.5 & 14.6 & 12.7 & 0.45 & 54.9 & 56.6 & 22.2 & 1.02 & 124.8 & 99.9 \\
\hline MZC0017 & 3.1 & 0.54 & 0.53 & 1.9 & 18.3 & 1.95 & 11.8 & 46.8 & 21.4 & 1.75 & 29.8 & 51.9 \\
\hline MZC0018 & 7.2 & 2.10 & 17.4 & 21.9 & 19.0 & 1.94 & 34.9 & 64.3 & 26.9 & 1.93 & 56.3 & 87.7 \\
\hline MZC0019 & 8.6 & 0.20 & 24.1 & 23.6 & 18.6 & -0.20 & 110.4 & 147.1 & 25.8 & 0.55 & 134.9 & 117.7 \\
\hline MZC0020 & 11.3 & -0.61 & 121.5 & 114.4 & 10.0 & 0.48 & 7.0 & 16.7 & 21.4 & 1.54 & 64.5 & 66.2 \\
\hline MZC0021 & 5.6 & 1.06 & 1.02 & 0.83 & 23.5 & 1.68 & 45.0 & 55.1 & 29.2 & 1.68 & 52.8 & 64.9 \\
\hline MZC0022 & 3.2 & 0.58 & 1.1 & 2.08 & 37.5 & 1.84 & 114.2 & 120.5 & 40.8 & 1.93 & 74.3 & 102.2 \\
\hline MZC0023 & 6.7 & 0.32 & 2.9 & 5.4 & 17.9 & 1.15 & 40.9 & 33.6 & 24.6 & 0.93 & 77.3 & 62.0 \\
\hline MZC0024 & 7.2 & 0.92 & 21.1 & 17.0 & 20.3 & 1.49 & 56.3 & 54.8 & 27.0 & 1.30 & 154.6 & 128.0 \\
\hline MZC0025 & 5.4 & 0.59 & 17.5 & 15.1 & 20.9 & 1.49 & 36.0 & 38.8 & 26.0 & 1.89 & 60.6 & 87.3 \\
\hline MZC0026 & 4.1 & 0.51 & 1.3 & 2.7 & 16.9 & 0.15 & 26.1 & 50.5 & 20.8 & 0.03 & 29.0 & 70.1 \\
\hline MZC0027 & 2.9 & 0.15 & 1.3 & 5.8 & 25.3 & 0.61 & 55.4 & 50.5 & 28.1 & 0.87 & 33.9 & 28.0 \\
\hline MZC0028 & 12.6 & 2.19 & 6.1 & 14.3 & 20.8 & 0.24 & 12.4 & 33.5 & 33.4 & 0.45 & 38.2 & 45.5 \\
\hline MZC0029 & 6.8 & -1.07 & 67.4 & 82.3 & 28.6 & 1.88 & 39.6 & 63.2 & 35.5 & 1.70 & 204.4 & 187.1 \\
\hline MZC0030 & 2.9 & 0.61 & 0.79 & 1.7 & 12.8 & 0.13 & 10.1 & 39.5 & 15.6 & 0.26 & 16.4 & 39.9 \\
\hline MZC0031 & 5.2 & 0.46 & 3.25 & 4.5 & 17.7 & 1.08 & 84.2 & 67.6 & 22.9 & 0.83 & 112.3 & 91.2 \\
\hline
\end{tabular}


Table 4 continued

\begin{tabular}{|c|c|c|c|c|c|c|c|c|c|c|c|c|}
\hline & \multicolumn{4}{|c|}{ Storage root yield } & \multicolumn{4}{|c|}{ Vine yield } & \multicolumn{4}{|c|}{ Biomass } \\
\hline & $\mathrm{x}_{\mathrm{i}}$ & $\beta$ & MS & Eco & $\mathrm{x}_{\mathrm{i}}$ & $\beta$ & MS & Eco & $\mathrm{x}_{\mathrm{i}}$ & $\beta$ & MS & Eco \\
\hline MZC0032 & 5.2 & 1.61 & 9.73 & 10.2 & 34.9 & 1.94 & 4.6 & 40.1 & 40.1 & 1.56 & 11.7 & 24.8 \\
\hline MZC0033 & 6.0 & 1.38 & 6.47 & 6.2 & 15.3 & 0.14 & 1.1 & 31.6 & 20.4 & 0.01 & 30.6 & 73.2 \\
\hline MZC0034 & 7.7 & 2.18 & 17.3 & 23.1 & 19.3 & 0.97 & 114.3 & 91.5 & 26.0 & 1.21 & 204.9 & 166.1 \\
\hline MZC0035 & 2.5 & 0.29 & 0.65 & 3.9 & 16.9 & 0.98 & 3.8 & 3.1 & 19.4 & 0.92 & 8.20 & 6.9 \\
\hline MZC0036 & 4.6 & 0.64 & 17.9 & 15.2 & 10.8 & 1.20 & 20.8 & 18.3 & 15.3 & 1.55 & 51.8 & 56.1 \\
\hline MZC0037 & 7.0 & 1.12 & 17.4 & 14.0 & 22.7 & 1.31 & 4.4 & 7.6 & 29.7 & 1.13 & 25.2 & 21.0 \\
\hline MZC0038 & 6.6 & 1.14 & 7.7 & 6.3 & 27.1 & 1.65 & 43.8 & 52.5 & 33.7 & 1.60 & 30.8 & 41.9 \\
\hline MZC0039 & 5.4 & 0.23 & 11.3 & 13.0 & 13.4 & 1.16 & 59.5 & 48.7 & 18.8 & 1.29 & 98.0 & 82.6 \\
\hline MZC0040 & 3.9 & 0.73 & 8.1 & 7.0 & 23.3 & 2.34 & 133.2 & 180.5 & 27.1 & 1.63 & 192.6 & 173.6 \\
\hline MZC0041 & 6.9 & 1.82 & 5.3 & 8.6 & 17.4 & 1.51 & 23.1 & 29.0 & 24.3 & 1.55 & 35.6 & 43.2 \\
\hline MZC0042 & 7.5 & 0.13 & 4.2 & 8.4 & 26.0 & 0.28 & 71.3 & 78.7 & 32.9 & -0.16 & 85.4 & 134.3 \\
\hline MZC0043 & 11.9 & 1.98 & 60.1 & 54.4 & 16.2 & 1.06 & 29.5 & 23.7 & 28.1 & 1.53 & 133.9 & 121.0 \\
\hline MZC0044 & 4.1 & 0.79 & 2.1 & 2.0 & 24.2 & 0.13 & 186.3 & 180.5 & 29.3 & 0.12 & 219.1 & 213.7 \\
\hline MZC0045 & 2.5 & 0.44 & 1.26 & 3.1 & 7.6 & 0.55 & 13.3 & 19.0 & 10.1 & 0.66 & 9.0 & 13.0 \\
\hline MZC0046 & 7.5 & 1.49 & 19.5 & 17.2 & 34.3 & 2.74 & 40.0 & 157.1 & 41.0 & 2.61 & 122.5 & 226.3 \\
\hline MZC0047 & 6.2 & 0.51 & 10.2 & 9.7 & 9.8 & 0.40 & 18.1 & 29.3 & 16.4 & 0.63 & 29.8 & 30.5 \\
\hline MZC0048 & 6.8 & 2.23 & 15.9 & 22.7 & 7.0 & 0.04 & 11.8 & 47.6 & 14.2 & 0.17 & 88.5 & 104.5 \\
\hline MZC0049 & 6.0 & 1.64 & 10.2 & 10.9 & 8.3 & -0.02 & 28.1 & 65.2 & 14.6 & 0.12 & 86.8 & 107.6 \\
\hline MZC0050 & 3.6 & 1.23 & 6.7 & 5.7 & 7.9 & 0.47 & 4.1 & 14.8 & 10.4 & 0.15 & 32.7 & 61.2 \\
\hline MZC0051 & 6.6 & 2.57 & 24.3 & 35.9 & 8.0 & -0.12 & 21.5 & 69.0 & 13.7 & -0.34 & 151.6 & 210.2 \\
\hline MZC0052 & 5.1 & 0.87 & 1.5 & 1.3 & 9.1 & 0.35 & 3.0 & 19.6 & 14.2 & 0.52 & 3.6 & 14.0 \\
\hline MZC0053 & 8.3 & 2.15 & 28.1 & 31.3 & 23.2 & 1.42 & 35.2 & 35.5 & 30.7 & 0.66 & 17.1 & 19.5 \\
\hline MZC0054 & 9.5 & 3.04 & 59.5 & 75.1 & 11.0 & 0.90 & 7.5 & 6.4 & 20.5 & 0.56 & 131.0 & 114.6 \\
\hline MZC0055 & 2.5 & 0.56 & 3.6 & 4.1 & 12.1 & 0.29 & 8.3 & 27.1 & 14.6 & 0.44 & 12.2 & 25.3 \\
\hline MZC0056 & 4.3 & 0.79 & 14.9 & 12.2 & 9.6 & 0.03 & 64.3 & 89.9 & 13.8 & 0.07 & 75.4 & 102.8 \\
\hline MZC0057 & 6.5 & 1.91 & 9.9 & 13.3 & 9.0 & 0.17 & 33.9 & 55.8 & 15.5 & 0.34 & 128.2 & 123.9 \\
\hline MZC0058 & 2.1 & 0.47 & 3.5 & 4.7 & 8.6 & -0.11 & 48.7 & 90.0 & 11.0 & -0.17 & 64.2 & 118.6 \\
\hline $\mathrm{LSD}_{0.05}$ & & 1.88 & & & & 1.23 & & & & 1.49 & & \\
\hline$\beta$-test & & & $* *$ & & & & $* *$ & & & & + & \\
\hline
\end{tabular}

**** and + indicate highly significant at $P \leq 0.01$ and significant at $P \leq 0.05$ or $P \leq 0.10$, respectively

The $G \times E$ variation was split due to regression $(\beta)$ and deviation from the regression lines (MS) - a dynamic concept of stability- and the environmental variance $\left(\sigma_{i}^{2}\right)$ - static concept of stability (Tables 4 , 6). A stable sweetpotato cultivar or landrace should have a higher trait value than the mean of the population, a $\beta=1$, and a non-significant deviation from the regression (Eberhart and Russell 1966). High storage root yields appear to be associated with either high stability or instability. A $\beta$ about 1 indicates average responsiveness, but if associated with high mean trait value the genotype will be rated as having general adaptability, while it will be regarded as a sweetpotato having poor adaptability if showing a low mean trait value (Finlay and Wilkinson 1963). A $\beta$ significantly below 1 indicates a sweetpotato with better adaptation of to low-yielding environments, e.g. ADMARC for total storage root yield (Table 4). The $\mathrm{CV}$ is a static stability-i.e., homeostasis-because it measures the dispersion of the data set. Static stability is when a stable genotype tends to maintain constant yield across different environments and shows minimum environmental sensitivity. The smaller the CV, the closer the data of each environment around the 


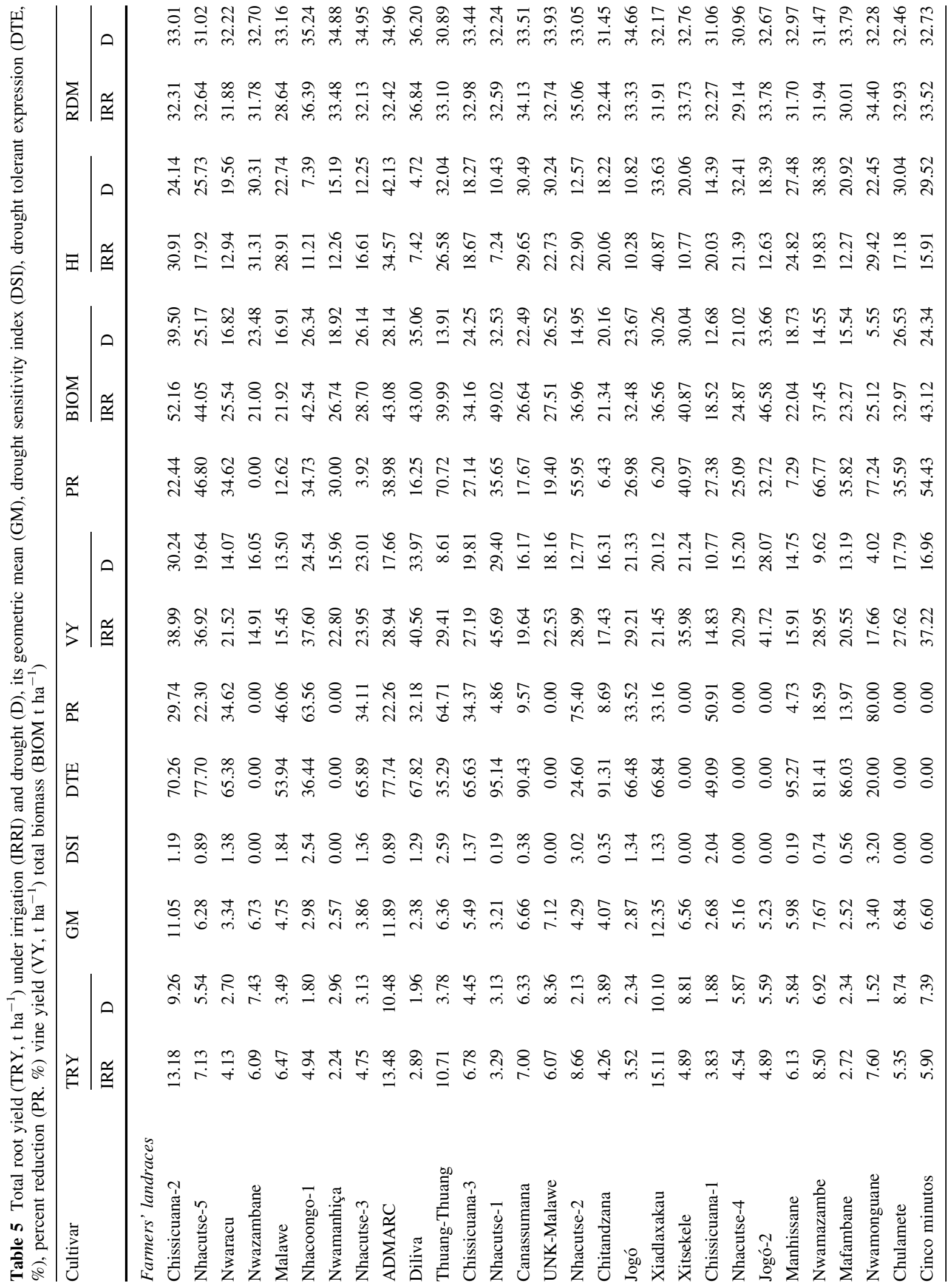




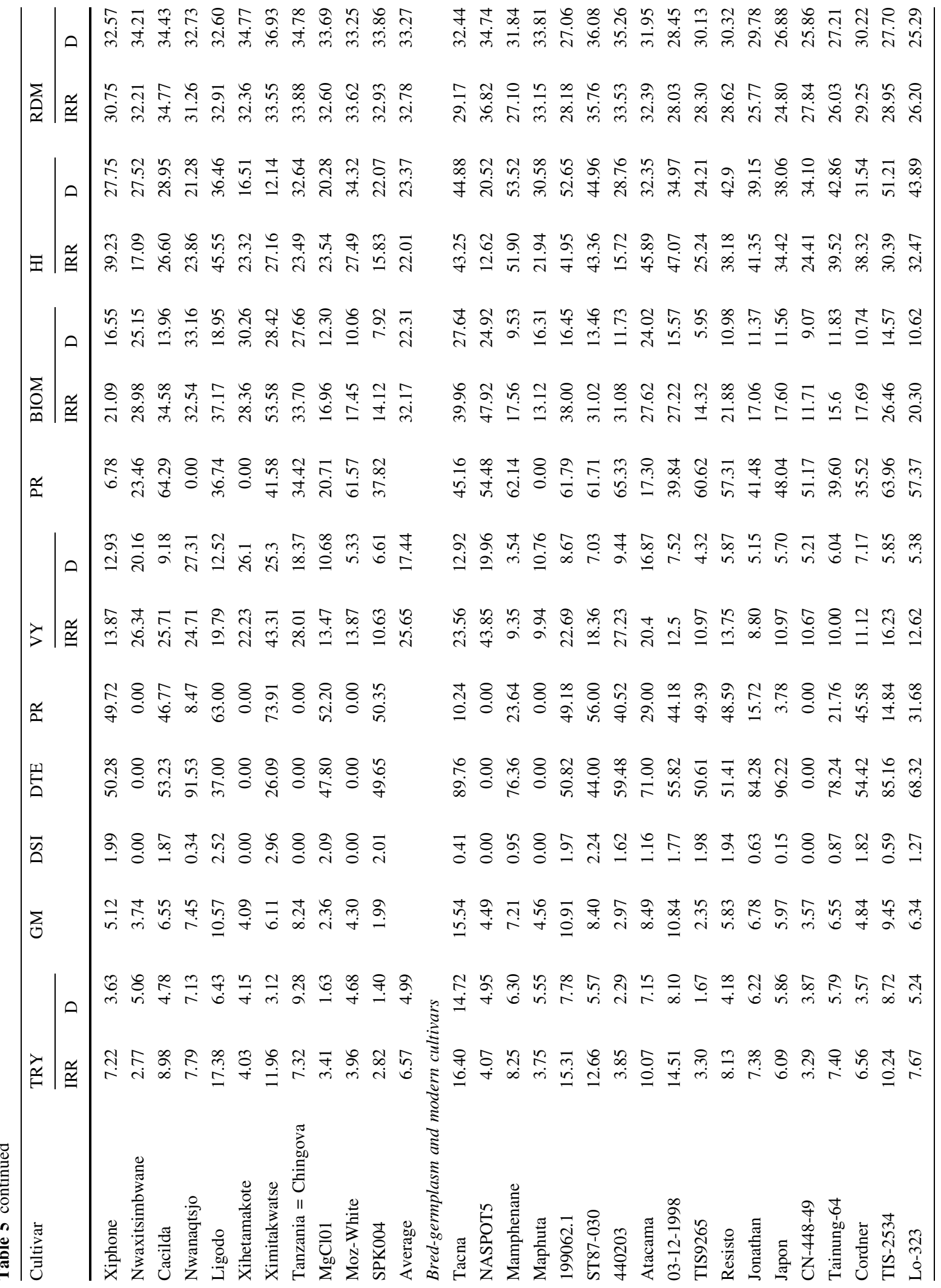




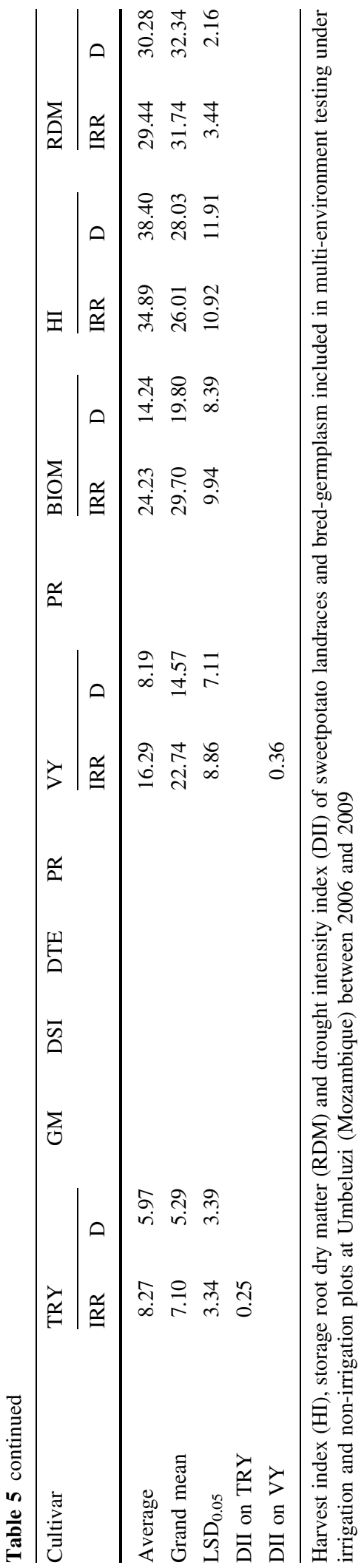

mean, and thus the more stable the genotype across the environments tested, e.g. ADMARC, Resisto, ST87030 and Xiphone for harvest index (Table 6). The ecovalence (Eco) is inversely related to phenotypic stability; i.e., a low Eco indicates high performance stability. However, sweetpotato clones with average to low storage yield had a low Eco, which suggests that genotypes with average to low edible yields lack a response to favorable environments, e.g. Nhacutse-5, 440203, Chissicuana-3 or Cordner. Selection based on low Eco may lead to sweetpotato-bred germplasm with low to average storage root yield. Combining statistic stability and performance may penalize high performance per se, as indicated before by Piepho (1994). Hence, storage root yield and static stability should be treated as two negatively associated traits when using a selection index.

The heterogeneity due to regression was only able to account for 10.9 and $16.8 \%$ the variance component due to $\mathrm{G} \times \mathrm{E}$ for storage root and biomass yield (Table 3), respectively, thus suggesting that AMMI analysis could provide further insights on the multienvironment trial data. In the AMMI bi-plots (Fig. 3) circles (black for irrigated and white for non-irrigated) were the symbols for the environments, while sweetpotato genotypes used black triangles. The first interaction principal component score (IPCA) accounted for $58.1 \%$ of the total treatment variation. The AMMI bi-plot shows the genotype and environment main effects along the abscissa, whereas the IPCA genotype and environment scores are on the ordinate. The vertical dotted line indicates the storage root yield grand mean $(\mu)$ whereas the horizontal line indicates a zero IPCA. Displacements along the abscissa and the ordinate show the differences in the main (additive) effects or the interaction effects, respectively. Chissicuana-2, ADMARC, Xiadlaxakau, Tacna and TIS-2534 had higher storage root yield than Resisto under irrigation and than Tanzania without irrigation. Chissicuana-2, ADMARC, Xiadlaxakau along with Tanzania, Nhacutse-5, Nwanaqtsjo, 199062.1, and TIS-2534 had also a storage root harvest above $4 \mathrm{t} \mathrm{ha}^{-1}$ even under extreme drought in 2008.

Harvest index and storage root yield were positively associated (Table 7), thus suggesting that selection for the former can be used to select high yielding sweetpotato bred-germplasm. Storage root yield was positively correlated with deviations from 
Fig. 2 Cluster analysis of 58 sweetpotato genotypes based on geometric yield $\left(\mathrm{t} \mathrm{ha}{ }^{-1}\right)$, drought sensitivity index (DSI), drought

tolerance expression (\%), percent reduction, harvest index $(\mathrm{HI})$ and the root dry matter $(\%)$
Dendrogram using Average Linkage (Between Groups)

Chulamet

Chingova

UNK-Malawe

Nwaxitsimbwane

Nhacutse-4

Nwamanhiça

Cinco minutos

NASPOT5

Xitsekele

Jogó-2

Moz-White

CN-448-49

Nwazambane

Maphuta

Xihetamakote

Jonathan

Tainung-64

Japon

Nwamazambe

TIS-2534

Mamphenane

Lo-323

Nhacutse-5

ADMARC

Tacna

Mafambane

Chissicuana-3

Jogó

Nwaracu

Nhacutse-3

Diliva

Xiadlaxakau

Atacama

Chissicuana-2

Chitandzana

Manhissane

Canassumana

Nwanactsjo

Nhacutse-1

199062.1

ST 87.030

Resisto

Cacilda

440203

TIS9265

12/3/998

Cordner

Malawe

Xiphone

Chissicuana-1

MgCl01

SPK004

Ligodo

Nhacoongo-1

Ximitakwatse

Thuang-Thuang

Nhacutse-2

Nwamonguane

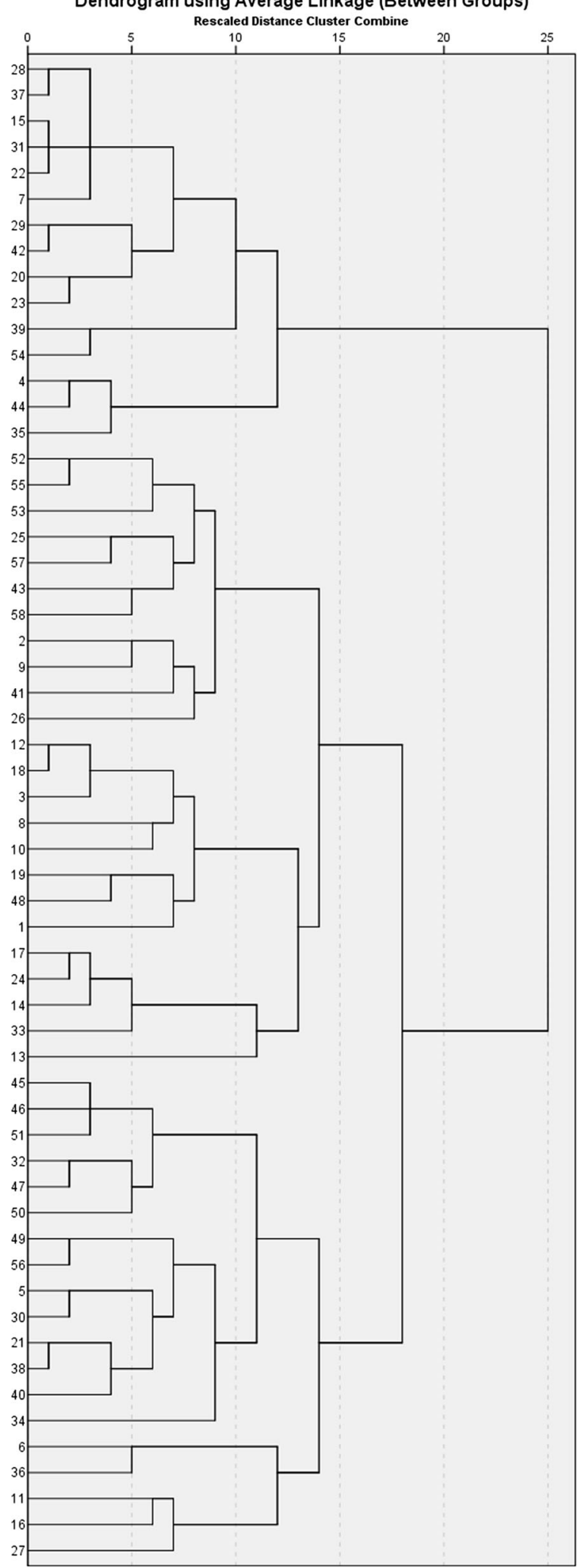


Table 6 Average $\left(\mathrm{x}_{\mathrm{i}}\right)$ harvest index and storage root dry matter content, their static concept of genotype $\times$ environment interaction (stability variance, $\sigma_{i}^{2}$; coefficient of variation, $\mathrm{CV} \%$ )

\begin{tabular}{|c|c|c|c|c|c|c|}
\hline \multirow[t]{2}{*}{ Cultivar } & \multicolumn{3}{|c|}{ Harvest index } & \multicolumn{3}{|c|}{ Storage root dry matter content } \\
\hline & $\mathrm{x}_{\mathrm{i}}$ & $\sigma_{i}^{2}$ & $\mathrm{CV}$ & $\mathrm{x}_{\mathrm{i}}$ & $\sigma_{i}^{2}$ & $\mathrm{CV}$ \\
\hline Tacna & 44.1 & 485.99 & 49.99 & 30.8 & 4.29 & 6.73 \\
\hline Chissicuana-2 & 27.5 & 379.96 & 70.88 & 32.7 & 7.99 & 8.64 \\
\hline Nhacutse-5 & 21.8 & 209.00 & 66.32 & 31.8 & 1.08 & 3.27 \\
\hline Nwaracu & 16.2 & 63.32 & 49.12 & 32.1 & 2.28 & 4.70 \\
\hline Nwazambane & 30.8 & 316.81 & 57.79 & 32.2 & 1.16 & 3.35 \\
\hline NASPOT5 & 16.6 & 245.84 & 94.45 & 35.8 & 2.97 & 4.81 \\
\hline Malawe & 25.8 & 228.87 & 58.64 & 30.9 & 10.72 & 10.60 \\
\hline Nhacoongo-1 & 9.3 & 15.30 & 42.06 & 35.8 & 3.27 & 5.05 \\
\hline Mamphenane & 52.7 & 699.70 & 50.19 & 29.5 & 8.66 & 9.98 \\
\hline Maphuta & 26.3 & 233.57 & 58.11 & 33.5 & 8.43 & 8.67 \\
\hline Nwamanhiça & 13.7 & 73.21 & 78.30 & 34.2 & 7.76 & 8.15 \\
\hline 199062.1 & 47.3 & 226.58 & 31.82 & 27.6 & 4.28 & 7.50 \\
\hline Nhacutse-3 & 14.4 & 91.41 & 66.40 & 33.5 & 3.28 & 5.41 \\
\hline ADMARC & 38.3 & 87.44 & 24.41 & 33.7 & 9.58 & 9.18 \\
\hline 15. Diliva & 6.1 & 15.49 & 64.52 & 36.5 & 11.23 & 9.18 \\
\hline ST87-030 & 44.2 & 82.68 & 20.57 & 35.9 & 2.93 & 4.77 \\
\hline 440203 & 22.2 & 246.45 & 70.71 & 34.4 & 2.20 & 4.31 \\
\hline Thuang-Thuang & 29.3 & 604.97 & 83.95 & 32.0 & 3.75 & 6.05 \\
\hline Atacama & 39.1 & 574.46 & 61.30 & 32.2 & 0.73 & 2.65 \\
\hline $1998-12-3$ & 41.0 & 527.39 & 59.90 & 28.2 & 0.82 & 3.21 \\
\hline Chissicuana-3 & 18.5 & 58.17 & 41.23 & 33.2 & 0.15 & 1.17 \\
\hline Nhacutse-1 & 8.8 & 34.11 & 66.37 & 32.4 & 2.63 & 5.01 \\
\hline Canassumana & 30.1 & 177.07 & 44.21 & 33.8 & 0.89 & 2.80 \\
\hline UNK-Malawe & 26.5 & 134.94 & 43.84 & 33.3 & 2.05 & 4.30 \\
\hline 25. Nhacutse-2 & 17.7 & 39.48 & 35.50 & 34.1 & 8.02 & 8.31 \\
\hline Chitandzana & 19.1 & 54.95 & 38.81 & 31.9 & 6.43 & 7.94 \\
\hline Jogó & 10.6 & 12.28 & 33.06 & 34.0 & 4.79 & 6.44 \\
\hline Xiadlaxakau & 37.3 & 178.45 & 35.81 & 32.0 & 2.72 & 5.16 \\
\hline Xitsekele & 15.4 & 145.60 & 78.35 & 33.2 & 5.54 & 7.09 \\
\hline Chissicuana-1 & 17.2 & 47.03 & 39.87 & 31.7 & 5.25 & 7.23 \\
\hline Nhacutse-4 & 26.9 & 256.66 & 59.56 & 30.1 & 14.15 & 12.50 \\
\hline 32. Jogó-2 & 15.5 & 219.66 & 95.62 & 33.2 & 5.46 & 7.04 \\
\hline Manhissane & 26.2 & 165.02 & 49.03 & 32.3 & 2.37 & 4.77 \\
\hline Nwamazambe & 29.1 & 257.25 & 55.12 & 31.7 & 0.45 & 2.12 \\
\hline Mafambane & 16.6 & 58.11 & 45.92 & 31.9 & 8.41 & 9.09 \\
\hline Nwamonguane & 25.9 & 89.02 & 36.43 & 33.3 & 12.52 & 10.62 \\
\hline Chulamete & 23.6 & 178.71 & 56.65 & 32.7 & 2.29 & 4.63 \\
\hline Cincominutos & 22.7 & 146.63 & 53.34 & 33.1 & 3.27 & 5.46 \\
\hline Xiphone & 33.5 & 85.49 & 27.60 & 31.7 & 7.76 & 8.78 \\
\hline Nwaxitsimbwane & 22.3 & 286.29 & 75.88 & 33.2 & 3.91 & 5.96 \\
\hline Cacilda & 27.8 & 349.62 & 67.26 & 34.6 & 2.85 & 4.88 \\
\hline Nwanaqtsjo & 22.6 & 67.58 & 36.38 & 32.0 & 2.58 & 5.02 \\
\hline Ligodo & 41.0 & 202.63 & 34.71 & 32.8 & 1.59 & 3.84 \\
\hline Xihetamakote & 19.9 & 310.50 & 88.55 & 33.6 & 2.54 & 4.74 \\
\hline
\end{tabular}


Table 6 continued

\begin{tabular}{|c|c|c|c|c|c|c|}
\hline \multirow[t]{2}{*}{ Cultivar } & \multicolumn{3}{|c|}{ Harvest index } & \multicolumn{3}{|c|}{ Storage root dry matter content } \\
\hline & $\mathrm{x}_{\mathrm{i}}$ & $\sigma_{i}^{2}$ & $\mathrm{CV}$ & $\mathrm{x}_{\mathrm{i}}$ & $\sigma_{i}^{2}$ & $\mathrm{CV}$ \\
\hline 45. TIS9265 & 24.7 & 118.15 & 44.01 & 29.2 & 6.09 & 8.44 \\
\hline Ximitakwatse & 19.6 & 271.81 & 84.12 & 35.2 & 8.32 & 8.19 \\
\hline Resisto & 40.5 & 87.47 & 23.09 & 32.0 & 2.01 & 4.83 \\
\hline Jonathan & 40.3 & 455.86 & 52.98 & 27.8 & 12.14 & 12.53 \\
\hline Japon & 36.2 & 252.51 & 43.90 & 25.8 & 4.04 & 7.79 \\
\hline CN-448-49 & 29.3 & 423.42 & 70.23 & 25.7 & 11.97 & 13.46 \\
\hline Tainung-64 & 41.2 & 557.01 & 57.28 & 26.6 & 17.18 & 15.58 \\
\hline Cordner & 34.9 & 277.01 & 47.69 & 29.7 & 5.94 & 8.21 \\
\hline Chingova & 28.1 & 595.41 & 86.84 & 34.3 & 4.48 & 6.17 \\
\hline TIS-2534 & 40.8 & 1057.29 & 79.70 & 28.3 & 1.52 & 4.36 \\
\hline $\mathrm{MgCl} 01$ & 21.9 & 112.24 & 46.39 & 33.1 & 4.50 & 6.41 \\
\hline Moz-White & 30.9 & 384.63 & 63.47 & 33.4 & 2.38 & 4.62 \\
\hline Lo-323 & 38.2 & 193.17 & 36.39 & 25.7 & 14.64 & 14.89 \\
\hline SPK004 & 19.0 & 199.61 & 74.36 & 33.4 & 4.27 & 6.19 \\
\hline
\end{tabular}

the regression, variances across environments and IPCAs from the AMMI (Table 7), thus indicating that storage root yield and its stability are inversely related among these sweetpotato landraces and modern cultivars. Storage root yield and harvest index also had the same correlation profiles with their trait stability (Table 7); i.e., a low $\mathrm{G} \times \mathrm{E}$ of storage root yield was associated with low $\mathrm{G} \times \mathrm{E}$ for harvest index. The fact that the harvest index was significantly associated to its variances across environments (Fig. 4; Table 7), which can be determined at an early breeding stage when using at least two environments, suggests the possibility on indirect selection for storage root yield and its stability through selecting simultaneously for a high harvest index with low environmental variance. In this way, sweetpotato cultivars with high root yield and stability may be bred, particularly when the heritability of harvest index is higher than that of storage root yield.

\section{Discussion}

Drought in these multi-year trials reduced significantly storage root yield by $25 \%$, which falls within the range (15-39\%), noticed previously after 20 consecutive days under this water deficit during the growing season (Gong and Wang 1990). Water shortage suppresses plant growth-which can be measured as vine yield in sweetpotato- due to loss of turgor in expanded cells (Kirnak et al. 2001). This reduced source strength negatively affected the amount of storage root yield under drought. Sweetpotato is regarded as moderately drought tolerant (Valenzuela et al. 2000) especially when the onset of the drought starts after the root initiation stages. In these multi-year trials, drought began at the middle of the root initiation phase, thereby leading to a moderate water shortage.

In case of irregular rains - as they occur in Mozambique and other countries of southern Africa- there is limited knowledge whether it is possible to breed for sweetpotato clones which are adapted to drought and respond to rains adequately. Knowledge on the $\mathrm{G} \times \mathrm{E}$ structure is therefore important to facilitate recommendations for cultivar releases and to make informed choices regarding selection of cultivars with specific or wide adaptation in sweetpotato breeding programs (Grüneberg et al. 2005). Storage root yield is influenced by various factors. The combined analysis of variance for storage root yield across year environments, genotypes and $\mathrm{G} \times \mathrm{E}$ interaction significantly affected the storage root yield of genotypes. The relative variance component for $\mathrm{G} \times \mathrm{E}$ was highest for storage root yield among the three measured traits. The significant $G \times$ E suggests that some of the genotypes were not stable between treatments and from year to year. Ranking of genotypes changed between 
Fig. 3 The additive main effect and multiplicative interaction model 1 (AMMI1) bi-plot of sweetpotato clones(black triangles; numbering as per last two digits of IIAM codes given in Table 1) evaluated for storage root yield (YLD) in irrigated (black circles) and nonirrigated (white circles) environments at Umbeluzi in Mozambique between 2006 and 2009

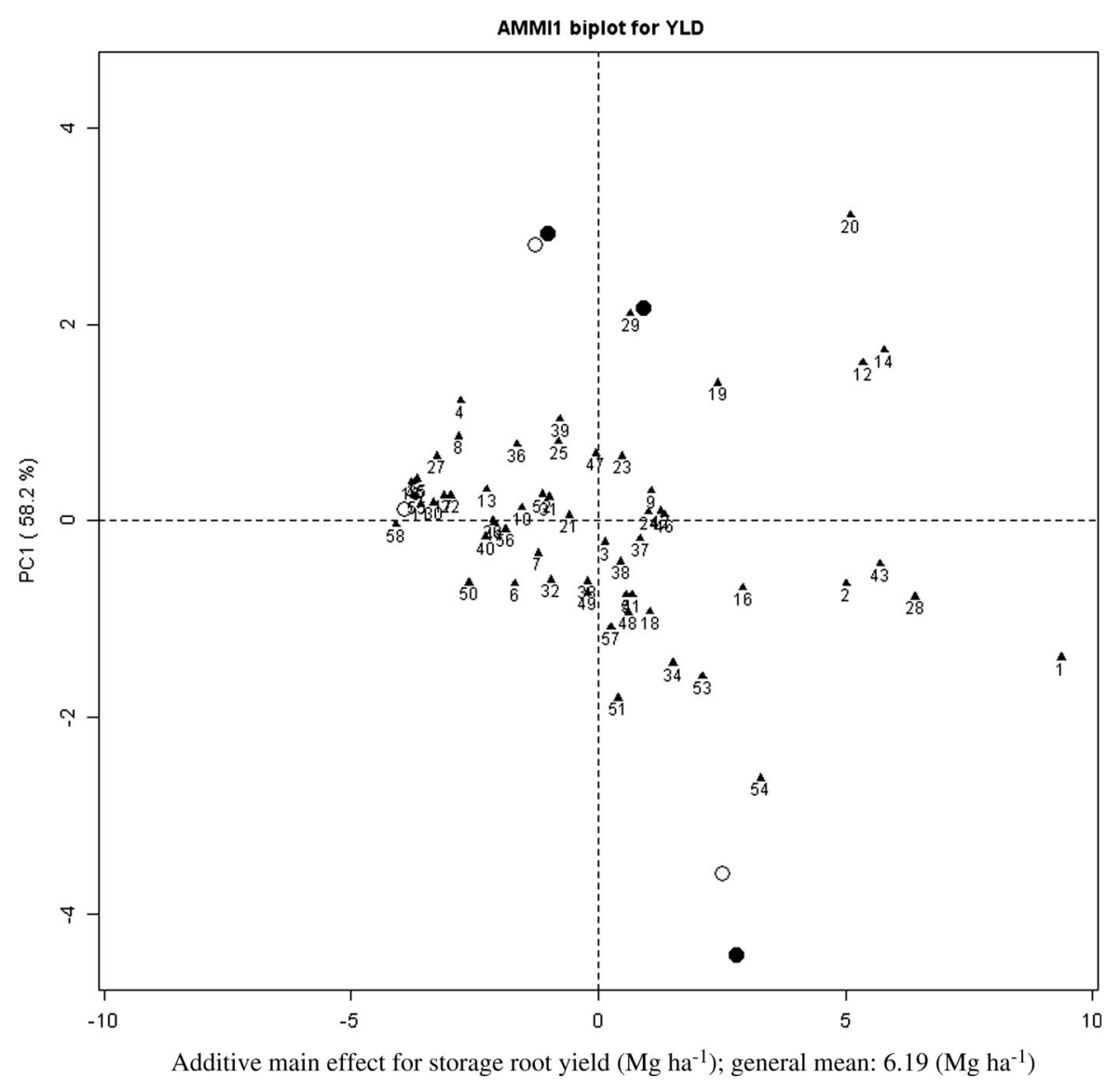

Table 7 Pearson correlation coefficients among means $\left(\mathrm{x}_{\mathrm{i}}\right)$, deviations from regression (Ms dev. R.), variances across environments $\left(\mathrm{V}_{\mathrm{env}}\right)$, and interactions principal component 1 (IPCA1) and 2 (PCA2) scores of the additive main effect multiplicative interaction (AMI) model for storage root yield and harvest index of sweetpotato landraces and bred-germplasm included in multi-environment testing under irrigation and non-irrigation plots at Umbeluzi (Mozambique) between 2006 and 2009

\begin{tabular}{|c|c|c|c|c|c|c|c|c|c|}
\hline & \multicolumn{6}{|c|}{ Storage root yield } & \multicolumn{3}{|c|}{ Harvest index } \\
\hline & $\mathrm{x}_{\mathrm{i}}$ & MS dev. R. & $\mathrm{V}_{\mathrm{env}}$ & IPCA1 & IPCA2 & $\mathrm{x}_{\mathrm{i}}$ & MS dev. R. & $\mathrm{V}_{\mathrm{env}}$ & IPCA1 \\
\hline \multicolumn{10}{|c|}{ Storage root yield } \\
\hline MS dev R. & $0.569 * * *$ & & & & & & & & \\
\hline $\mathrm{V}_{\text {env }}$ & $0.741 * * *$ & $0.802 * * *$ & & & & & & & \\
\hline IPCA1 & $0.532 * * *$ & $0.770 * * *$ & $0.785 * * *$ & & & & & & \\
\hline IPCA2 & $0.364 * *$ & $0.300 *$ & $0.363 * *$ & -0.053 & & & & & \\
\hline \multicolumn{10}{|c|}{ Harvest index } \\
\hline $\mathrm{x}_{\mathrm{i}}$ & $0.698 * * *$ & $0.403 * *$ & $0.576 * * *$ & $0.442 * *$ & 0.204 & & & & \\
\hline MS dev R. & $0.338 * *$ & $0.558 * * *$ & $0.546 * * *$ & $0.463 * *$ & 0.118 & $0.483 * * *$ & & & \\
\hline $\mathrm{V}_{\text {env }}$ & $0.416^{* *}$ & $0.428 * *$ & $0.659 * * *$ & $0.497 * * *$ & 0.195 & $0.574 * * *$ & $0.754 * * *$ & & \\
\hline IPCA1 & -0.119 & 0.227 & -0.175 & 0.080 & -0.140 & -0.127 & 0.064 & $-0.356^{* *}$ & \\
\hline IPCA2 & 0.248 & 0.031 & 0.215 & -0.077 & 0.232 & $0.356 * *$ & 0.088 & $0.268^{*}$ & -0.000 \\
\hline
\end{tabular}

****** and * indicate highly significant at $P \leq 0.001$ or $P \leq 0.01$, and significant at $P \leq 0.05$, respectively 


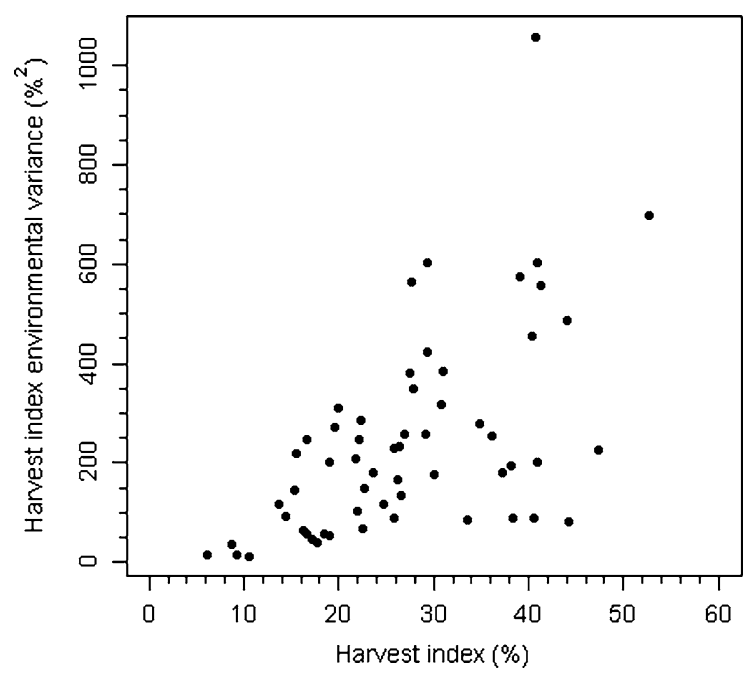

Fig. 4 Harvest index means and variance of harvest index across environments of sweetpotato clones at Umbeluzi in Mozambique between 2006 and 2009

treatments within a year and from year to year. $\mathrm{G} \times \mathrm{E}$ often poses a major challenge for cultivar selection in sweetpotato breeding.

Geometric mean and other drought indices are necessary for selection of sweetpotato genotypes performing well under both drought and optimum environments when the crossover $\mathrm{G} \times \mathrm{E}$ occurs. GMP, DTE and DSI identified ADMARC and Tacna as the two stable high-yielding performers. The harvest index of both-which belong to the same diversity cluster as per a recent analysis based on DNA markers (Maquia et al. 2013)—was high as well. These results indicate therefore that GMP, DTE, DSI and harvest index are useful to select genotypes that are high yielding under drought and optimum environments. Sweetpotato breeding programs have significantly improved storage root yield production of sweetpotato largely due to improved harvest index. The highest harvest index noticed in sweetpotato was $65 \%$ (Evans 1993; Gifford and Evans 1981). In these multi-year trial modern cultivars and other bredgermplasm had significantly higher storage root yield than the farmers' landraces. The former, however, did not show storage root yield stability as noticed by their higher percent reduction under drought than the farmers' landraces. This finding also suggests that sweetpotato breeding for drought adaptation was not a priority elsewhere.
In summary, sweetpotato production environments vary highly and selection on sweetpotato germplasm based on storage root yield alone under optimum environments would bring a disadvantage for farmers growing this crop in poor environments. The use of drought indices and harvest index look promising for selecting bred-germplasm for various production environments and their use would be encouraged to start early in the breeding cycle.

Acknowledgments The United States Agency for International Development (USAID), The Rockefeller Foundation, Bill \& Melinda Gates Foundation and the Alliance for a Green Revolution in Africa (AGRA) provided grant funding for this research.

Open Access This article is distributed under the terms of the Creative Commons Attribution 4.0 International License (http:// creativecommons.org/licenses/by/4.0/), which permits unrestricted use, distribution, and reproduction in any medium, provided you give appropriate credit to the original author(s) and the source, provide a link to the Creative Commons license, and indicate if changes were made.

\section{References}

Agili S, Nyende B, Ngamau K, Masinde P (2012) Selection, yield evaluation, drought tolerance indices of orange-flesh sweet potato (Ipomoea batatas Lam) hybrid clone. J Nutr Food Sci 2:3. doi:10.4172/2155-9600.1000138

Andrade M, Barker I, Cole D, Dapaah H, Elliott H, Fuentes S, Grüneberg W, Kapinga R, Kroschel J, Labarta R, Lemaga B, Loechl C, Low J, Lynam J, Mwanga R, Ortiz O, Oswald A, Thiele G (2009) Unleashing the potential of sweetpotato in sub-Saharan Africa: current challenges and way forward. Working paper 2009-1. International Potato Center, Lima, Perú

Anselmo BA, Ganga ZN, Badol EO, Heimer YM, Nejidat A (1998) Screening sweetpotato for drought tolerance in the Philippine highlands and genetic diversity among selected genotypes. Trop Agric 75:189-196

Becker HC, Leon J (1988) Stability analysis in plant breeding. Plant Breeding 101:1-23

Blum A (2005) Drought resistance, water-use efficiency, and yield potential-are they compatible, dissonant, or mutually exclusive? Aust J Agric Res 56:1159-1168

Bouwkamp JC (1985) Production requirements. In: Bouwkamp JC (ed) Sweetpotato products: a natural resource for the tropics. CRC Press, Boca Raton, pp 9-33

Caliskan ME, Erturk E, Sogut T, Boydak E, Arioglu H (2007) Genotype $\times$ environment interaction and stability analysis of sweetpotato (Ipomoea batatas) genotypes. N Z J Crop Hort Sci 35:87-99

Chávez R, Mendoza H, Mahesh U, Espinoza J, Cabello R, Arévalo N, Wijntje A, Scoffield J, Zúñiga P, Guevara E, 
Siles P (2000) Genetic improvement and adaptation of sweetpotato (Ipomoea batatas) to dry and saline conditions. IDESIA 18:97

Ding CW, Niu FX, Guo XD, Hua XX (1997) Identification on the drought resistance in sweetpotato genetic resources. J Henan Agric Sci 10:3-5

Eberhart SA, Russell WA (1966) Stability parameters for comparing varieties. Crop Sci 6:36-40

Ekanayake IJ (1990) Evaluation of potato and sweet potato genotypes for drought resistance. CIP Research Guide 19. International Potato Center. Lima, Perú

Evans LT (1993) Crop evolution, adaptation and yield. Cambridge University Press, Cambridge

FAO (2004) Drought impact mitigation and prevention in the Limpopo River Basin: a situation analysis. Land and water discussion paper. Food and Agriculture Organization of the United Nations, Rome, Italy http://ftp.fao.org/agl/aglw/ docs/lwdp4_e.pdf. Accessed 1 Sept 2012

FAO (2015) FAOSTAT. Food and Agriculture Organization of the United Nations, Rome, Italy, http://faostat.fao.org/ Accessed 9 Oct 2015

Finlay K, Wilkinson GN (1963) The analysis of adaptation in plant breeding program. Aust J Agric Res 14:742-754

Fischer R, Maurer R (1978) Drought resistance in spring wheat genotypes I. Grain yield responses. Aust J Agric Res 29:897-912

Fischer KS, Wood G (1981) Breeding and selection for drought tolerance in tropical maize. In: Proceedings of the symposium. Principles and methods in crop improvement for drought resistance with emphasis on rice. Los Baños, Philippines, pp 23-25, May 1981International Rice Research Institute Los Baños Philipiness

Gauch HG (1992) Statistical analysis of regional yield trials: AMMI analysis of factorial designs. Elsevier, Amsterdam

Gifford RM, Evans LT (1981) Photosynthesis, carbon partitioning, and yield. Annu Rev Plant Physiol 32:485-509

Gollob HF (1968) A statistical model which combines features of factor analytic and analysis of variance techniques. Psychometrika 33:73-114

Gong Y, Wang G (1990) An investigation on the effect of drought stress on growth of sweet potato and measures to improve drought resistance and stabilize yields. Zhejiang Agric Sci 1:26-29

Grüneberg WJ, Manrique K, Zhang D, Hermann M (2005) Genotype $\times$ environment interactions for a diverse set of sweetpotato clones evaluated across varying ecogeographic conditions in Peru. Crop Sci 45:2160-2171

Henderson SA, Sitch LA, Macoo P, Botão M, Pequenino F, Whyte J (1997) Multilocation trials of sweetpotato varieties in Northern and Central Mozambique. Roots: newsletter of the Southern Africa root crops research network (SARRNET) and the East Africa root crops research network (EARRNET) 4:1. International Institute of Tropical Agriculture, Ibadan, Nigeria

Hou LX, Xiao LZ, Kang ZH, Yang XP, Gu JH, Tang BJ, Liu JB (1999) Identification on drought resistance of sweetpotato varieties. J Henan Agric Sci 2:5-6

Indira P, Kabeerathumma S (1988) Physiological response of sweet potato under water stress. 1: effect of water stress during different phases of tuberization. J Root Crops $14: 37-40$
Jannsens M (1983) Genotype by environment interactions of the yield components in sweet potato. In: Shideler FS, Rincón $\mathrm{H}$ (eds) Proceedings of the sixth symposium international society Tropical Root Crops, Lima, Perú. 21-26 Feb 1983. International Potato Center, Lima, Perú, pp 543-551

Kelm M, Brück H, Hermann M, Sattelmacher B (2000) Plant productivity and water use efficiency of sweetpotato (Ipomoea batatas) as affected by nitrogen supply. CIP program report 1999-2000. International Potato Center, Lima, Perú

Kirnak H, Kaya C, Tas I, Higgs D (2001) The influence of water deficit on vegetative growth, physiology, fruit yield and quality in eggplants. Bulg J Plant Physiol 27:34-46

Kivuva BM, Githiri SM, Yencho GC, Sibiya J (2014) Genotype $\times$ environment interaction for storage root yield in sweetpotato under managed drought stress conditions. J Agric Sci 6:41-56

Kottek M, Grieser J, Beck C, Rudolf B, Rubel F (2006) World map of the Köppen-Geiger climate classification updated. Meteorol Z 15:259-263

Maquia I, Muocha I, Naico A, Martins N, Gouveia M, Andrade I, Goulao LF, Ribeiro AI (2013) Molecular, morphological and agronomic characterization of the sweet potato (Ipomoea batatas L.) germplasm collection from Mozambique: genotype selection for drought prone regions. S Afr J Bot 88:142-151

MICOA (2005) Avaliação da vulnerabilidade as mudanças climáticas e estratégias de adaptação. Ministério Para a Coordenação da Acção Ambiental, Maputo

Monneveux P, Belhassen E (1996) The diversity of drought adaptation in the wide. Plant Growth Regul 20:85-92

Nasayao LZ, Saladaga FA (1998) Genotype $\times$ environment interaction for yield in sweetpotato. Philipp J Crop Sci 13:99-104

Piepho HP (1994) A comparison of the ecovalence and the variance of relative yield as measures of stability. J. Agron Crop Sci 173:1-4

R Core Team (2015) R: a language and environment for statistical computing. R foundation for statistical computing, Vienna, Austria. https://www.R-project.org/

Ramirez-Vallejo P, Kelly JD (1998) Traits related to drought resistance in common bean. Euphytica 99:127-136

Rukundo P, Shimelis H, Laing M, Gahakwa D (2013) Storage root formation, dry matter synthesis, accumulation and genetics in sweetpotato. Aust J Crop Sci 7:2054-2061

SAS Institute, Inc. (1988) SAS/STAT user's guide, release 6.03. SAS Institute, Inc. Cary, North Carolina

SAS Institute, Inc. (1997) SAS/STAT user's guide, release 6.12. SAS Institute, Inc. Cary, North Carolina

Tekalign T (2007) Genotype $\times$ environment interaction for root yield of elite sweet potato (Ipomoea batatas (L.) Lam.) genotypes. S Afr J Plant Soil 24:144-146

Thiyagu D, Rafii MY, Mahmud TMM, Latif MA, Malek MA, Sentoori G (2013) Genotype by environment assessment in sweetpotato as leafy vegetable using AMMI model. Pak J Bot 45:843-852

Utz HF (1997) PLABSTAT. Ein Computerprogram zur statistichen Analyse von pflanzenzuchterischen Experiment. Version 2B. Insititut fur Planzenzuchtung, Saatgutforschung and Populationsgenetik, Universitatt Hohenheim, Germany 
Valenzuela H, Fukuda S, Arakaki A (2000) Sweetpotato production guidelines for Hawaii. http://www.extension. hawaii.ed/kbase/reports/sweetpot.prod.htm. Accessed 1 Sept 2012

Wang YP, Liu QC, Li AX, Zhai H, Zhang SS, Liu BL (2003) In vitro selection and identification of drought-tolerant mutants in sweetpotato. Agric Sci China 2:1314-1320

Wricke G (1962) IJber eine Methode zur Erfassung der okologischen Streubreite in Feldversuchen. Z Pflanzenziicht 47:92-96

www.portaldogoverno.gov.mz/docs_gov/estrategia/agricultura/ Avaliacao_vulnerab_mud_climat_estrateg_adapt.pdf. Accessed 1 Sept 2012

Yang GH, Kang ZH, Xiao LH, Hou LX, Yang XP, Gu JH (1999) Breeding of a new starch processing type sweetpotato variety Yushu 13 with high yield and multi-resistance. J Henan Agric Sci 3:3-5
Yen CT, Chu CV, Sheng CL (1964) Studies on the drought resistance of sweetpotato varieties. Crop Sci (China) 3:183-190

Zhang MS, Xie B, Tan F, Zhang QT (2003) Relationship among soluble protein, chlorophyll and ATP in sweetpotato under water stress with drought resistance. Sci Agric Cínica 36:13-16

Zhang MS, Peng ZH, Xie B, Tan F, Zhang QT, Fu YF, Yang CX, Yang YH (2004) Relationship between water loss rate of cutting leaves and osmotic regulators under water stress and drought resistance in sweetpotato. Sci Agric Cínica 37:152-156

Zhang J, Jia W, Yang J, Abdelbagi MI (2006) Role of ABA in integrating plant responses to drought and salt stresses. Field Crops Res 97:111-119 